\title{
Phase Plane Analysis Method of Nonlinear Traffic Phenomena
}

\author{
Wenhuan Ai, Zhongke Shi, and Dawei Liu \\ College of Automation, Northwestern Polytechnical University, Xian, Shaanxi 710072, China \\ Correspondence should be addressed to Zhongke Shi; shizknwpu@126.com
}

Received 1 April 2015; Accepted 21 May 2015

Academic Editor: Hung-Yuan Chung

Copyright (C) 2015 Wenhuan Ai et al. This is an open access article distributed under the Creative Commons Attribution License, which permits unrestricted use, distribution, and reproduction in any medium, provided the original work is properly cited.

\begin{abstract}
A new phase plane analysis method for analyzing the complex nonlinear traffic phenomena is presented in this paper. This method makes use of variable substitution to transform a traditional traffic flow model into a new model which is suitable for the analysis in phase plane. According to the new model, various traffic phenomena, such as the well-known shock waves, rarefaction waves, and stop-and-go waves, are analyzed in the phase plane. From the phase plane diagrams, we can see the relationship between traffic jams and system instability. So the problem of traffic flow could be converted into that of system stability. The results show that the traffic phenomena described by the new method is consistent with that described by traditional methods. Moreover, the phase plane analysis highlights the unstable traffic phenomena we are chiefly concerned about and describes the variation of density or velocity with time or sections more clearly.
\end{abstract}

\section{Introduction}

It is really necessary to study various traffic phenomena for ultimately alleviating and preventing traffic jams. Only when the formation mechanism and inherent law of the traffic phenomena are investigated comprehensively can we organize the traffic scientifically, maximize the true potential of existing traffic network, reduce economic losses, and obtain the desired effect eventually.

In the last several decades, a lot of traffic flow phenomena were discovered on highways and urban expressways all over the world, such as traffic breakdown, hysteresis effect, synchronized flow, stop-and-go traffic, wide moving jam, traffic bottlenecks, shock wave, and rarefaction wave. Among them, shock wave, rarefaction wave and stop-and-go traffic have recently become the mainstream of research. The shock wave theory held that shock waves may form when the state is very different from the others in neighboring regions, and the shock front is the position of changing traffic state. Some researchers have described shock wave and rarefaction wave with the use of new models they proposed [1-3]. Ban et al. [4] have calculated the length of the vehicle queue according to the shock wave in traffic flow. The phenomenon of stopand-go in traffic flow has also got the particular attention by many researchers in the past decades. In 1971, Payne [5] has described a typical nonequilibrium state of stop-and-go phenomenon by using a high order continuous model he proposed. Kerner and Konhäuser also studied the structure of stop-and-go waves and the characteristic parameters of them [6]. They found that the parameters of stop-and-go waves do not depend on the amplitude of the perturbation but only on the parameters of the flow. Zhang and Shen investigated how parameters and the geometry of a lane-drop site may affect the stop-and-go traffic patterns by numerical calculation [7]. Some researchers analyzed the complicated stop-and-go phenomenon through the new traffic flow models proposed by them $[2,3,8,9]$. The results are consistent with the diverse nonlinear dynamical phenomena observed in the freeway traffic.

Various analysis methods of the transport phenomena also emerged in the past decades. Some scholars evaluated traffic phenomena by means of observation and controlled experiments and have made abundant achievements [10-12]. Parallel with experiments, many physical models have been proposed with the aim of describing the traffic phenomena and optimizing traffic flow. Due to the analogy of vehicle stream with gas stream and fluid stream, many of the macro continuum models of traffic flow have been developed. On the basis of LWR model [13, 14], a number of highorder continuum models have been presented to overcome 
the deficiencies of the LWR model. Jiang et al. [2] presented a new continuum model that does not have the characteristic speed problem. Tang et al. [15] put forward a new macro model for traffic flow with the consideration of the driver's forecast effect. Peng et al. [16] presented a new macro model of traffic flow considering the anticipation optimal velocity. Besides, some microscopic models of driver-vehicle behavior have also been proposed, which include the early linear models proposed by Chandler et al. [17], the early nonlinear models presented by Pipes [18] and many recent remarkable works of Wang et al. [19], Zhou et al. [20], Tang et al. [21], Hu et al. [22], and so on. They treat each individual vehicle as a particle and regard traffic as a complex system of interacting particles. Based on these models, researchers can use a lot of system simulation methods to analyze the traffic phenomena.

However, these methods cannot completely describe the complex traffic flow phenomena in phase plane from a system stability perspective. In this paper, we introduce a completely different method to analyze the complex nonlinear traffic phenomena. The variable substitution is adopted to transform a traditional traffic flow model into a new model which is suitable for the analysis in phase plane. From the phase plane diagrams, we can see that the traffic congestion corresponds to the unstable system. So the traffic flow problems can be converted into the system stability problems. In order to illustrate this new method further, some famous traffic phenomena such as stop-and-go waves, shock wave, and rarefaction wave are also discussed in this paper. When the stop-and-go traffic appears and the traffic flow fluctuations tend to be unstable, the curves in phase plane diagrams are divergent and many of them tend to infinity. When the shock wave and rarefaction wave appears, the curves in phase plane diagrams change from one equilibrium point to another.

The remainder of the paper is organized as follows. In Section 2, we present the description of variable substitution and get a new traffic flow model through variable substitution based on Jiang's model [2]. In Section 3, we analyze the well-known stop-and-go phenomena using the phase plane diagrams based on the new model and compare with the traditional temporal evolution of vehicle density. In Section 4, the shock and rarefaction waves are also studied to illustrate this new method further. We conclude the paper in Section 5.

\section{Variable Substitution Based on Jiang's Model}

Jiang et al. [2] proposed a continuum model, which has been mostly studied nowadays and has the following form:

$$
\begin{aligned}
\frac{\partial \rho}{\partial t}+\frac{\partial(\rho v)}{\partial x} & =0 \\
\frac{\partial v}{\partial t}+v \frac{\partial v}{\partial x} & =a\left[V_{e}(\rho)-v\right]+c_{0} \frac{\partial v}{\partial x}
\end{aligned}
$$

where $\rho$ is the density; $v$ is the velocity; $x$ and $t$ represent space and time, respectively; $a$ is the driver's sensitivity which equals the inverse of the driver's reaction time; $c_{0}$ represents the propagation speed of the disturbance; and $V_{e}[\rho(x, t)]$ is the optimal velocity function and has the following form [6]:

$$
\begin{aligned}
& V_{e}[\rho] \\
& \quad=v_{f}\left\{\left[1+\exp \left(\frac{\rho / \rho_{m}-0.25}{0.06}\right)\right]^{-1}-3.72 \times 10^{-6}\right\} .
\end{aligned}
$$

Here $v_{f}$ is the free-flow speed and $\rho_{m}$ is the maximum or jam density.

In the present paper, a simple transformation is employed as follows:

$$
\begin{aligned}
& \sigma=\frac{1}{v} \\
& \eta=\frac{1}{\rho_{m}-\rho} .
\end{aligned}
$$

Substituting the variables into (1), we have a new traffic flow model as follows:

$$
\begin{array}{r}
\sigma^{2} \frac{\partial \eta}{\partial t}+\left(\eta-\rho_{m} \eta^{2}\right) \frac{\partial \sigma}{\partial x}+\sigma \frac{\partial \eta}{\partial x}=0, \\
\frac{\partial \sigma}{\partial t}+\left(\frac{1}{\sigma}-c_{0}\right) \frac{\partial \sigma}{\partial x}+a \sigma\left(\sigma v_{e}(\eta)-1\right)=0 .
\end{array}
$$

Similarly, substituting the variables into (2), the equilibrium velocity $V_{e}[\eta]$ is as follows:

$$
\begin{aligned}
& V_{e}(\eta) \\
& \quad=v_{f}\left\{\left[1+\exp \left(\frac{0.75-1 / \eta \rho_{m}}{0.06}\right)\right]^{-1}-3.72 \times 10^{-6}\right\} .
\end{aligned}
$$

Applying the finite difference method to discretize the model, the state variables $\eta$ and $\sigma$ can be solved out. According to the variable substitution $\sigma=1 / \nu$, we can see that as long as the traffic becomes congested and the vehicles velocity goes to zero, the state variable $\sigma$ will approach infinity. Likewise, from the variable substitution $\eta=1 /\left(\rho_{m}-\rho\right)$ we can see that if the vehicle density is close to the jam density $\rho_{m}$, the state variable $\eta$ will approach infinity in the same way. Hence we may conclude that as long as the value of $\eta$ or $\sigma$ increases, the vehicle density will increase, the mean velocity will decrease, and the traffic system will tend to be unstable. Moreover, as long as the value of $\eta$ or $\sigma$ approaches infinity, there will be traffic jam formation and the traffic system will become unstable. The more the value of $\eta$ or $\sigma$ increases, the greater the fluctuation of the vehicle density is and the more unstable the traffic system is. On the contrary, the system becomes more stable. So the system instability could be reflected by the two variables. Furthermore, the variation range of the state variables $\rho$ and $v$ could be enlarged to infinity through such variable substitutions. To be specific, the variation range of $\rho$ in the original traffic flow model is $0-0.2 \mathrm{veh} / \mathrm{m}$ and the variation range of $v$ is $0-30 \mathrm{~m} / \mathrm{s}$, so there are rather limited changes in the diagrams about these variables. When the traffic becomes congested, the state variables $\rho$ and $v$ both 
tend to a specific value. However, if we substitute the state variables $\eta$ and $\sigma$ for $\rho$ and $v$, the variation range of both state variables $\eta$ and $\sigma$ will increase to infinity. As long as the traffic has a small fluctuation, the value of $\eta$ or $\sigma$ will change sharply. Moreover, as long as there is traffic jam formation, the value of $\eta$ or $\sigma$ will approach infinity. The traffic jams can be directly equated to the system instability in the phase plane. Thus we can use the phase plane diagrams about the variables $\eta$ or $\sigma$ to describe clearly the relationship between traffic jams and system instability.

\section{Analysis of the Stop-and-Go Traffic Phenomenon Using the New Method}

The stop-and-go traffic phenomenon is an international wellknown nonlinear phenomenon. Traditional researches on it mainly focused on using the figures of temporal development of density [2, 3, 6-9]. The new model mentioned above can also describe it through the phase plane diagrams from a system stability perspective. The comparisons and discussions between the two methods by using models (1) and (4) were given as follows.

The stop-and-go phenomena can be observed in the amplification of a small disturbance. Let us consider the behavior of a localized perturbation, which at time $t=0$ occurred in an initial homogeneous state of traffic flow and was given by [4]

$$
\begin{aligned}
& \rho(x, 0)=\rho_{0}+\Delta \rho_{0}\left\{\cosh ^{-2}\left[\frac{160}{L}\left(x-\frac{5 L}{16}\right)\right]\right. \\
& \left.-\frac{1}{4} \cosh ^{-2}\left[\frac{40}{L}\left(x-\frac{11 L}{32}\right)\right]\right\} \quad x \in[0, L], \\
& v(x, 0)=V(\rho(x, 0)) \quad x \in[0, L],
\end{aligned}
$$

where $\rho_{0}$ is the initial vehicle density, $\Delta \rho_{0}=0.01 \mathrm{veh} / \mathrm{m}$ is the amplitude of localized perturbation, and $L=32.2 \mathrm{~km}$ is the length of road section under consideration. The dynamic approximate boundary condition was given by

$$
\begin{aligned}
& \rho(1, t)=\rho(2, t), \\
& \rho(L, t)=\rho(L-1, t), \\
& v(1, t)=v(2, t), \\
& v(L, t)=v(L-1, t) .
\end{aligned}
$$

For computational purpose, the space domain was divided into equal intervals of length $100 \mathrm{~m}$ and time interval was chosen as $1 \mathrm{~s}$. The related parameters of our model were as follows:

$$
\begin{aligned}
c_{0} & =6 \mathrm{~m} / \mathrm{s}, \\
\tau & =14 \mathrm{~s}, \\
v_{f} & =30 \mathrm{~m} / \mathrm{s}, \\
\rho_{m} & =0.2 \mathrm{veh} / \mathrm{m}, \\
\Delta \rho_{0} & =0.01 \mathrm{veh} / \mathrm{m} .
\end{aligned}
$$

The critical density values of model (1) corresponding to the parameters above were $0.031 \mathrm{veh} / \mathrm{m}$ and $0.084 \mathrm{veh} / \mathrm{m}$, which can easily be found out by the stability condition [4]. The traffic flow will be unstable between these critical densities. The small disturbance in these initial homogeneous conditions will be amplified, and the stop-and-go phenomena will occur. Taking different values of initial density we explored what the unstable and stable traffic will evolve into.

Using model (4), we can obtain the numerical solution of $\eta$ and $\sigma$ by applying the forward difference method. Then we can draw four phase plane diagrams. The coordinate systems of them are $(\eta, \partial \eta / \partial t),(\eta, \partial \eta / \partial x),(\sigma, \partial \sigma / \partial t)$, and $(\sigma, \partial \sigma / \partial x)$, respectively. Through the four graphs the variation of density or velocity with time or sections can be investigated more clearly. Thus we can completely convert the fluctuations of traffic flow into the stability analysis charts.

Figure 1 shows the unstable traffic situation with small perturbations divergence when the initial density $\rho_{0}$ was set to $0.046 \mathrm{veh} / \mathrm{m}$ and the amplitude of localized perturbation $\Delta \rho_{0}$ was set to $0.01 \mathrm{veh} / \mathrm{m}$. Figure $1(\mathrm{a})$ is the density temporal evolution of the model (1). Since the value of initial density we set was in the unstable range, the amplitude of the initial small perturbations grows in time, leading to traffic instability. A complex localized structure consisting of two or more clusters forms. This situation corresponds to stop-and-go traffic.

Figures 1(b)-1(e) are the phase plane diagrams of model (4). First, a cross-sectional analysis was made along a time axis of Figure 1(a) to observe the variation of density with time when the section is fixed. Since the state variable $\eta$ increases strictly monotonously with the density, the change rule curves of $\eta$ with time on each road section are shown in Figure 1(b). We drew the curves in the figure one by one in the order of road section and found the curve of each downstream section moved toward the outer ring of upstream section. It demonstrated that density fluctuations were gradually amplified toward the upstream section. The initial small perturbations spread upstream and were divergent, leading to the whole traffic system instability.

The phase plane diagrams emphasized the instable situation of the traffic system we are chiefly concerned about. It can be seen that the value of $\eta$ will change sharply in Figure 1(b) even when there is very small fluctuation in Figure 1(a). When the vehicle density approached congestion, the value of $\eta$ will get larger. The corresponding curves of such traffic jams accounted for a large proportion in the graphs while most small amplitude density fluctuations accounted for a quite small percentage and they just centered in a small area near the initial value.

Figure 2 is the change curves of density and $\eta$ on the fiftieth road section which changes along with time. It can be seen from Figure 2(a) that there were many density fluctuations at this road section in the first ten minutes and this situation corresponds to stop-and-go traffic.

It reflects the density fluctuations outstandingly in Figure 2(b). We find out the starting point of the curve to observe its trajectory. It moves from the innermost point to the outermost circle and then turns toward the small circles inside. A circle of the phase plane diagram corresponds to a density fluctuation of the density curve chart. The variation 


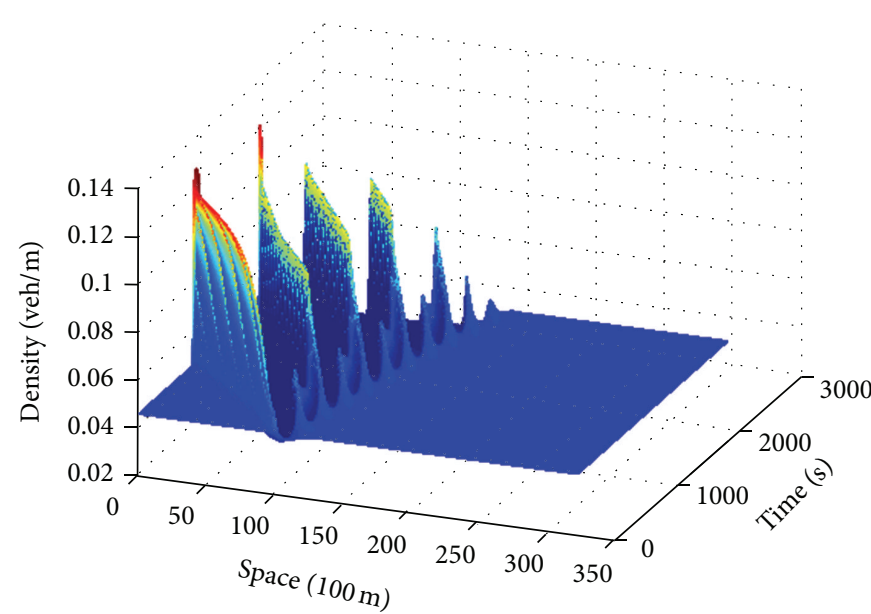

(a)

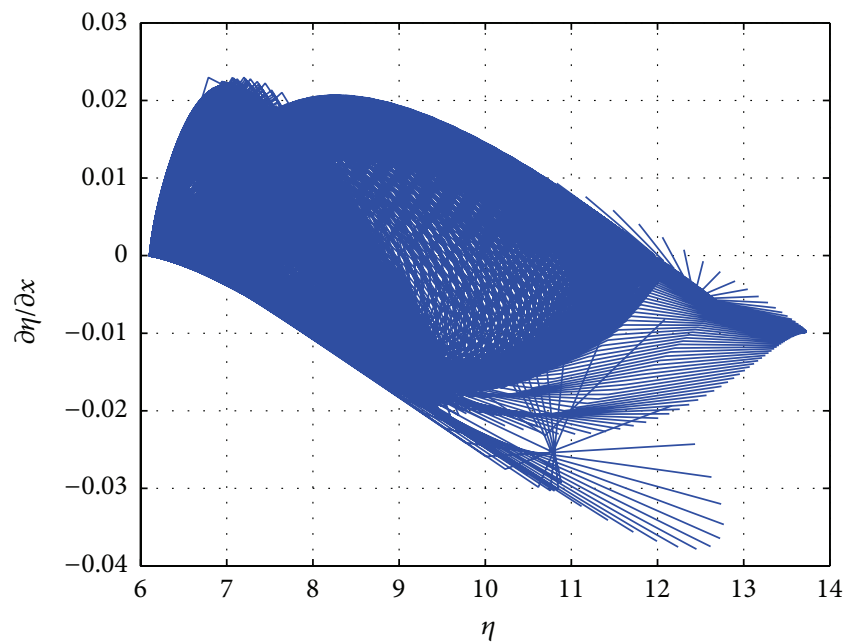

(c)

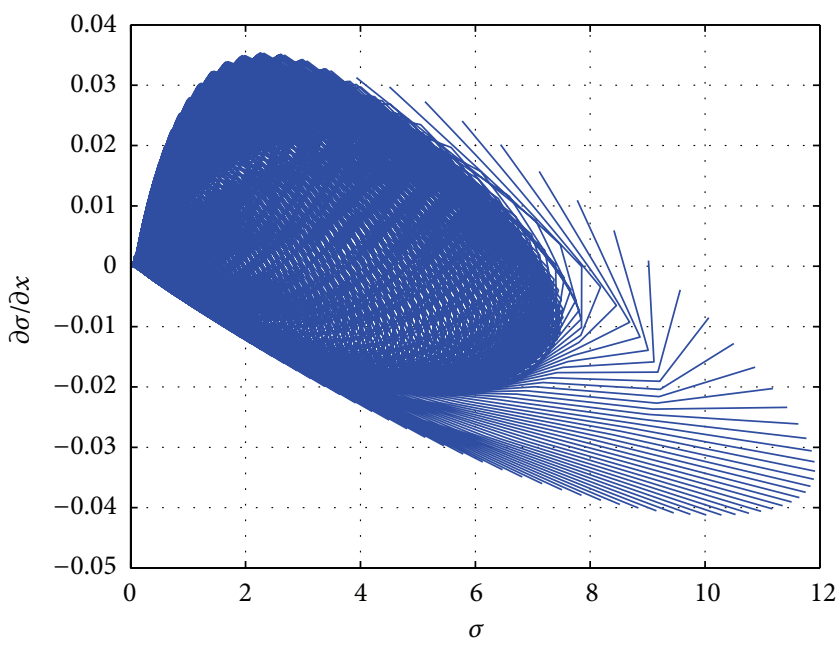

(e)

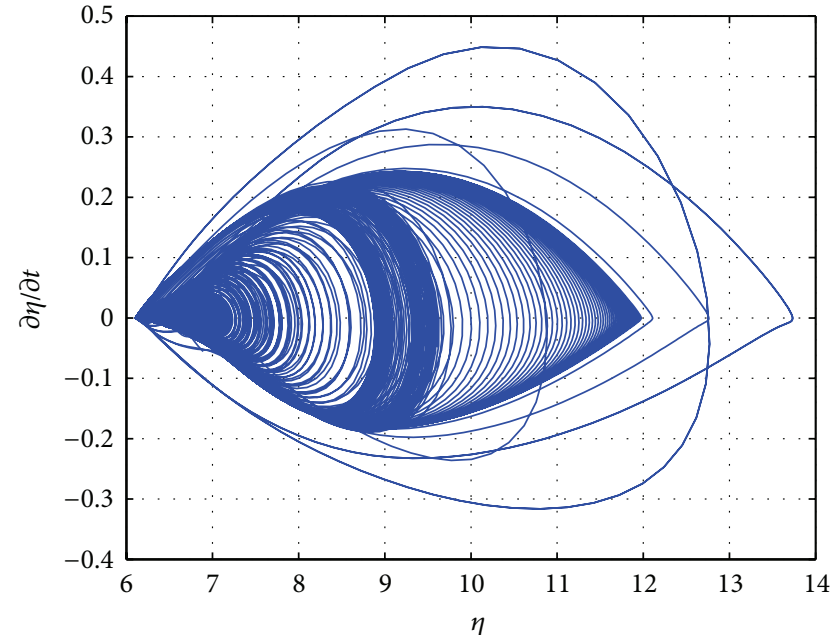

(b)

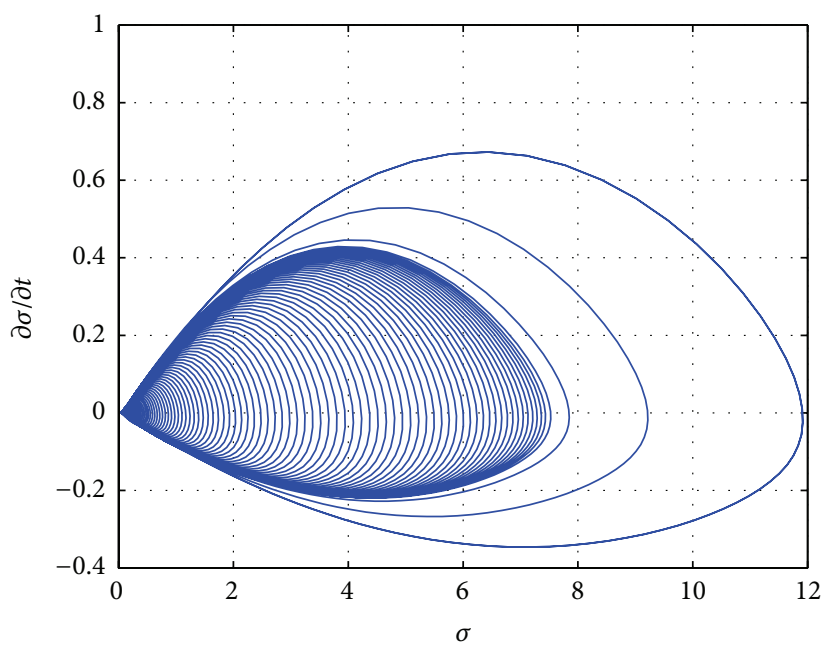

(d)

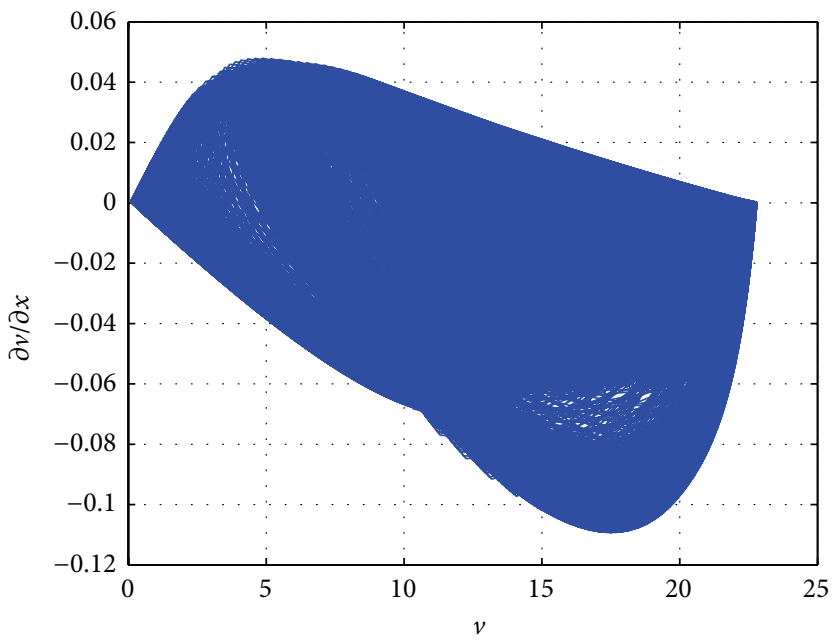

(f)

FIGURE 1: Evolution of small perturbation under the initial density $\rho_{0}=0.046 \mathrm{veh} / \mathrm{m}$ : (a) the temporal evolution of vehicle density; (b) the phase plane diagram of $(\eta, \partial \eta / \partial t)$; (c) the phase plane diagram of $(\eta, \partial \eta / \partial x)$; (d) the phase plane diagram of $(\sigma, \partial \sigma / \partial t)$; (e) the phase plane diagram of $(\sigma, \partial \sigma / \partial x)$; (f) the phase plane diagram of $(v, \partial v / \partial x)$. 


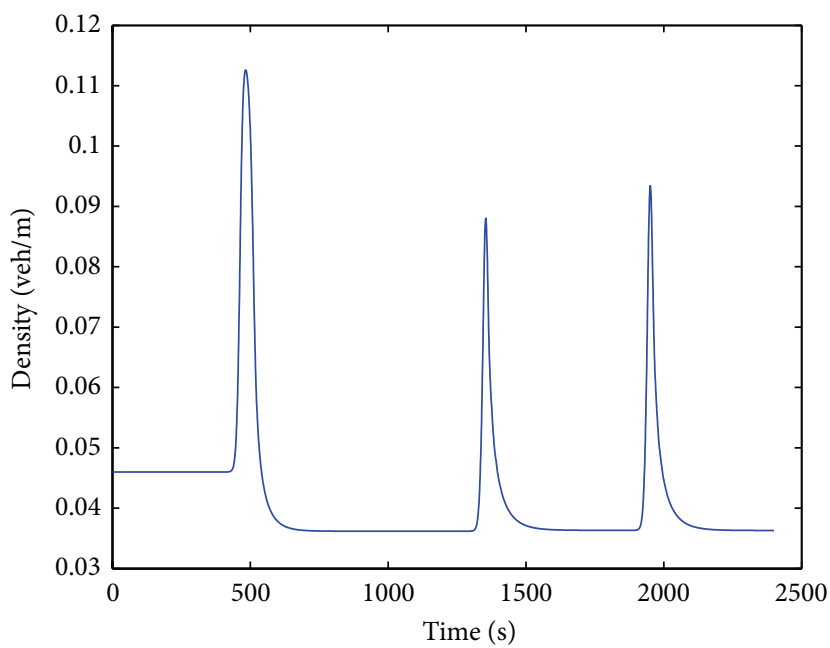

(a)

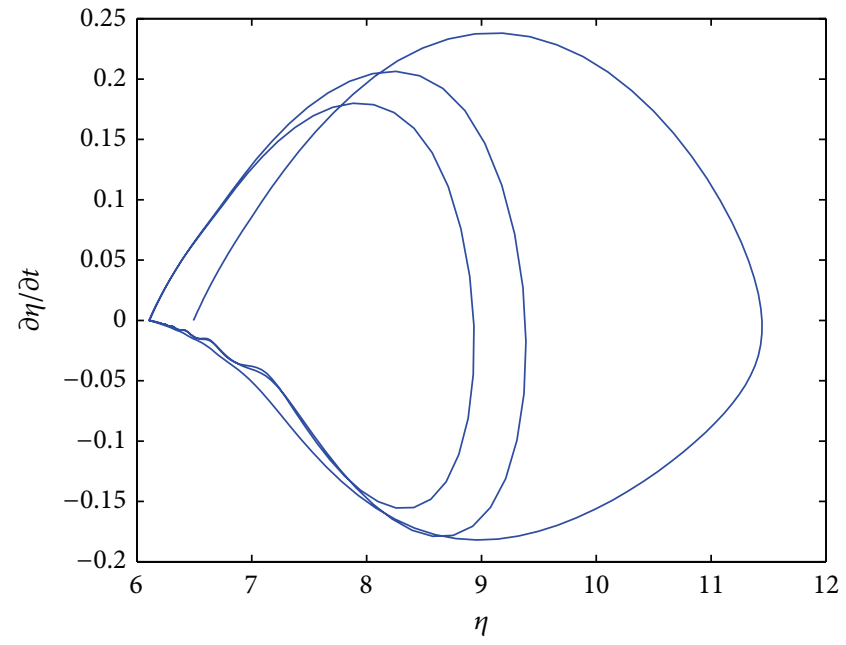

(b)

Figure 2: (a) The density-time variation curve on the fiftieth road section; (b) the phase plane diagram of $(\eta$, $\partial \eta / \partial t)$ on the fiftieth road section.

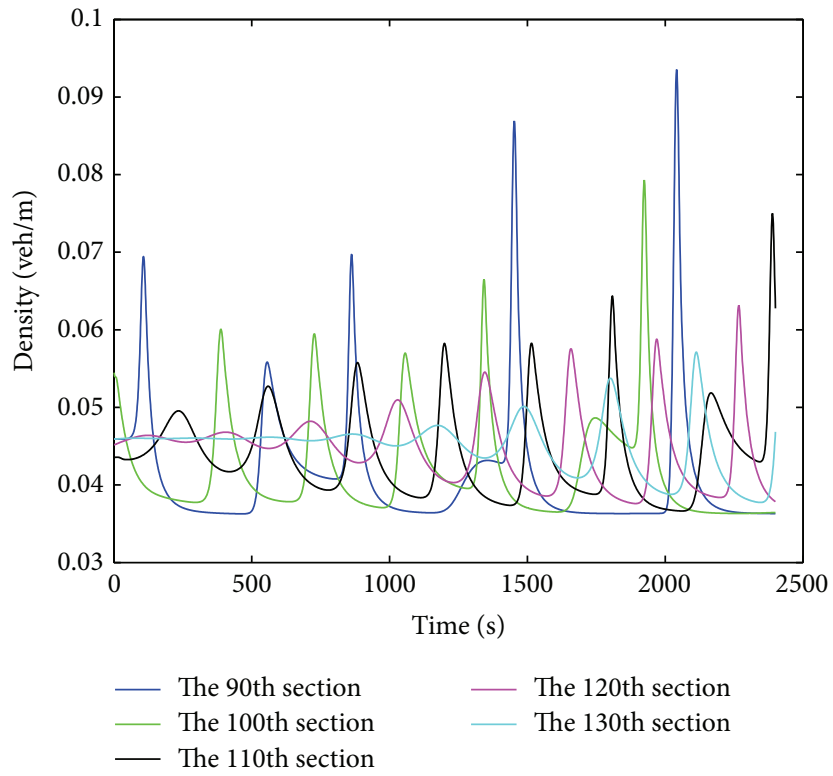

(a)
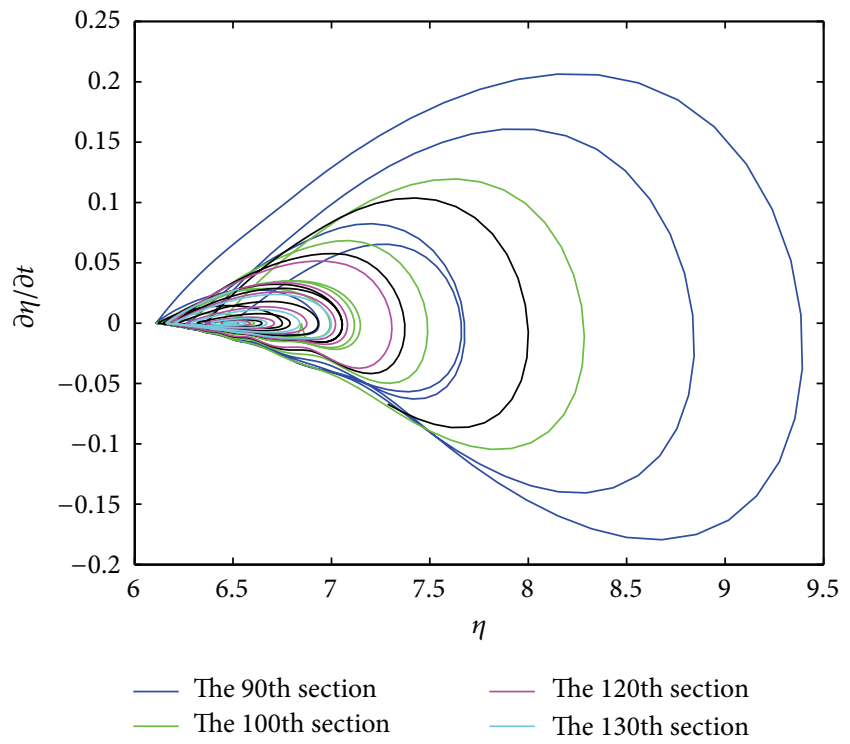

(b)

FIGURE 3: (a) Density-time variation curves on the 130th, the 120th, the 110th, the 100th, and the 90th road section; (b) the phase plane diagram of $(\eta, \partial \eta / \partial t)$ on the 130th, the 120th, the 110th, the 100th, and the 90th road section.

of the circle ring in horizontal direction corresponds to the amplitude of a density fluctuation and it in vertical direction corresponds to the change rate of a density fluctuation. So the phase plane diagrams clearly reflect the size and speed of the density fluctuations. This is consistent with the stop-andgo traffic phenomenon described by the density curve chart. Moreover, it mainly reflects the unstable traffic conditions we are chiefly concerned with.

Figure 3 is the change curves of density and $\eta$ in the first 40 minutes on the 130th, the 120th, the 110th, the 100th, and the 90th road section which change along with time. As you can see from Figure 3(a), the amplitude of the density fluctuation on the 130th section is small, as shown by the cyan line. It is growing toward the upper section of the road because the initial small perturbations spread upstream and are divergent. In Figure 3(b), the variation of $\eta$ on the 130th section is small, as shown by the cyan circle inside. It grows toward the upper sections and reaches the largest on the 90th section as shown by the blue circle outside. So it also shows that the initial perturbation is amplified upstream. Although 

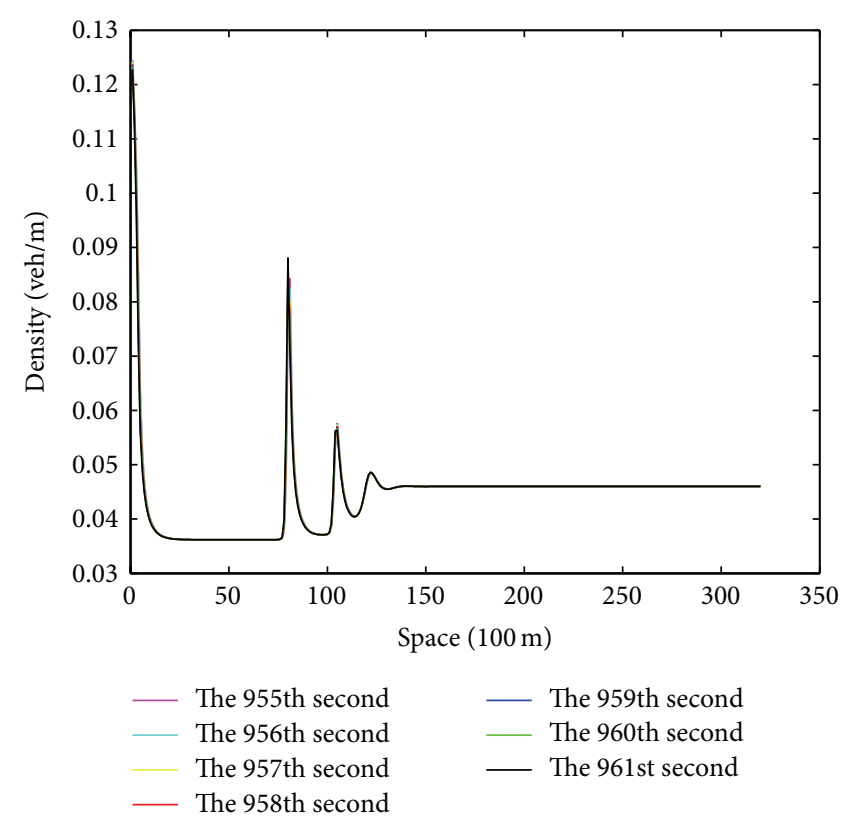

(a)

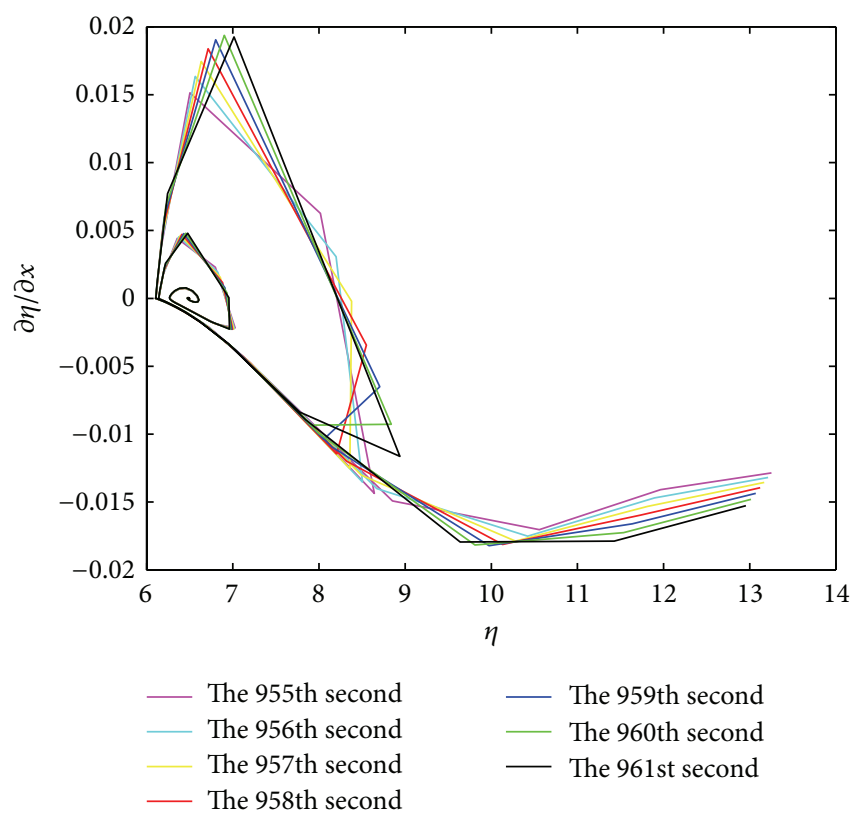

(b)

Figure 4: (a) Density-space variation curves on the 955th, the 956th, the 957th, the 958th, the 959th, the 960th, and the 961st second on the whole road section; (b) the phase plane diagrams of $(\eta, \partial \eta / \partial x)$ on the 955th, the 956th, the 957th, the 958th, the 959th, the 960th, and the 961st second of the whole road section.

there are very small density fluctuations in Figure 3(a), the variation range of $\eta$ is big in Figure 3(b). The phase plane diagram mainly reflects the density fluctuations.

Similar to Figure 1(b), Figure 1(d) is the combination of variation curves of $\sigma$ on each road section during the first 40 minutes. Since $\sigma$ is inversely proportional to the vehicle velocity, it also describes the variation of velocity with time on each road section. If the traffic approaches congestion, the velocity will be close to zero. The state variable $\sigma$ will tend to infinity and the system will become unstable. We also found the curve of each downstream section expanded toward the outer ring of upstream section if we drew the curves one by one in the order of road section. It demonstrated that the average velocity gradually reduced toward the upstream section. The nearer from the upstream, the more volatile the whole traffic system is. Those curves describing such unstable traffic also account for a large proportion in the graph.

Then we made a cross-sectional analysis along a displacement axis of Figure 1(a) to observe the variation of density with displacement when time is fixed. Since the state variable $\eta$ increases strictly monotonously with the density, the change rule curves of $\eta$ per second on the whole road are shown in Figure 1(c). If we drew these curves one by one in the order of time, we can see all the curves change with time from the inner circle to the outer ring and many of them tend to infinity. It also demonstrates that density fluctuations of the whole road are gradually amplified with time. The amplitude of the initial small perturbations grows in time. The whole traffic system is unstable. Compared with the temporal evolution of density, the phase plane diagrams can more clearly reflect the density variation of current time and the next time.
Figure 4(a) is the density variation curves of the 955 th, the 956th, the 957th, the 958th, the 959th, the 960th, and the 961st second on the whole road section. The curves almost coincide together and we cannot see the obvious changes. Figure 4(b) is the phase plane diagrams corresponding to these times and it can clearly show that the curves enlarge per second from inside to outside; that means the amplitude of the density on the whole road grows in time and the traffic system is divergent.

Similar to Figure 1(c), Figure 1(e) could be considered as a group of curves which describe the changes of $\sigma$ per second on the whole road. Since $\sigma$ is inversely proportional to the vehicle velocity, it also describes the fluctuations of velocity per second on the whole road. If the traffic approaches congestion, the velocity will be close to zero. The state variable $\sigma$ will tend to infinity, the curves will be divergent, and the system will become unstable. In Figure 1(e), we can see many curves tend to infinity. So it illustrates intuitively that the amplitude of the initial small perturbations grows in time. So through the phase plane diagrams of $(\sigma, \partial \sigma / \partial x)$ we can convert directly the traffic jam phenomenon into the curves of instability system. Figure 1(f) is the phase plane diagram of $(v, \partial v / \partial x)$. It describes the changes of $v$ per second on the whole road. Although it reflects the velocity fluctuations, it could not focus on the traffic jam phenomena. We can see it more obviously by comparing the velocity variation of current time and the next time.

Figure 5(a) is the velocity-space variation curves of the 910th, 911th, the 912th, the 913th, the 914th, the 915th, and the 916th second on the whole road section. All of the curves almost coincide together and we cannot see the time variation of velocity. Figure 5(b) is the corresponding phase plane 


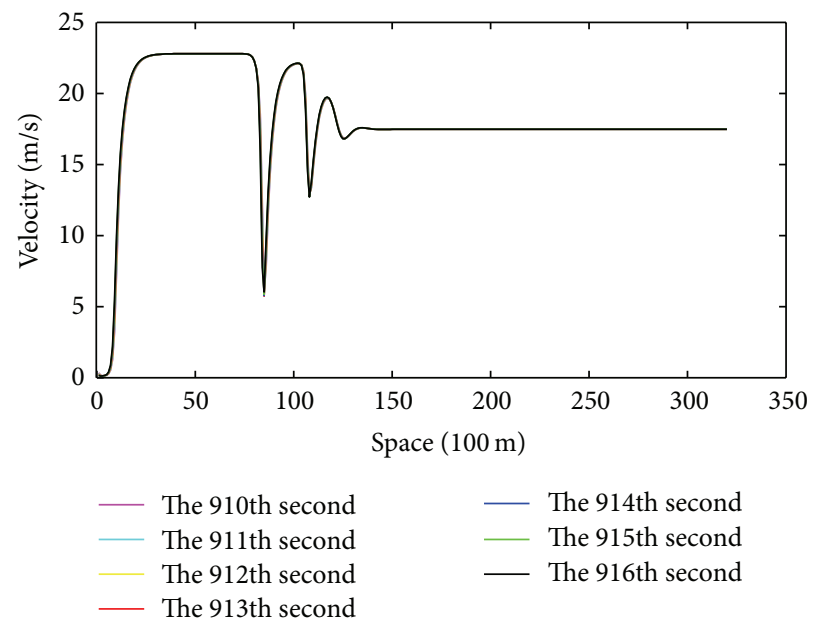

(a)

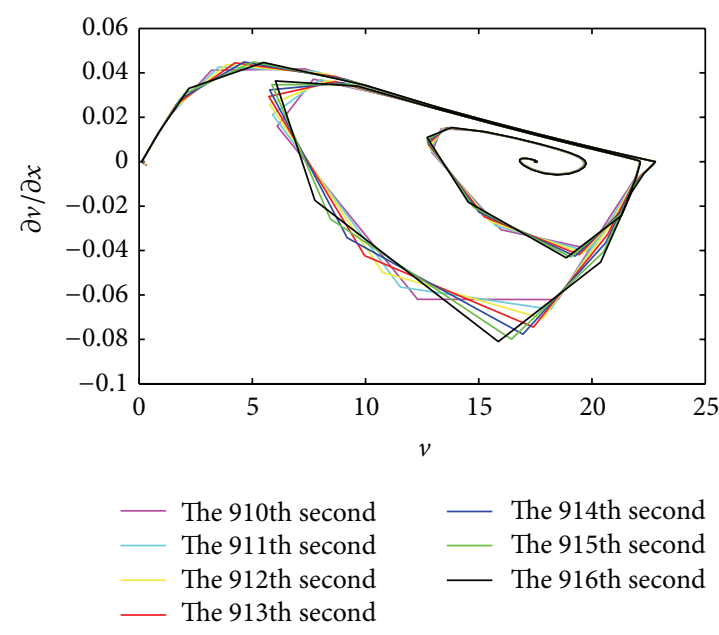

(b)

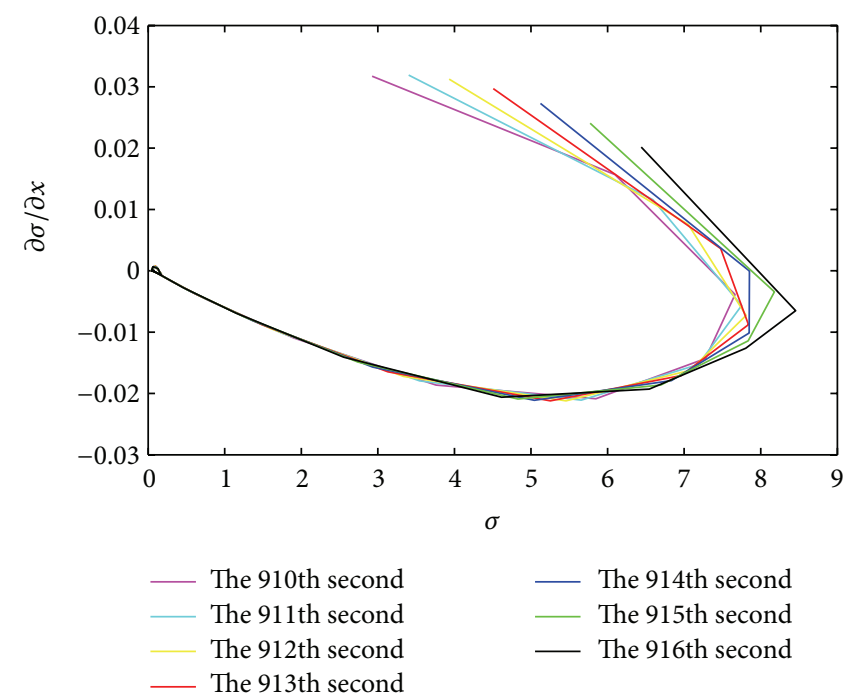

(c)

Figure 5: (a) Velocity-space variation curves on the 910th, 911th, the 912th, the 913th, the 914th, the 915th, and the 916th second of the whole road section; (b) the phase plane diagrams of $(v, \partial v / \partial x)$ on the 910th, 911th, the 912th, the 913th, the 914th, the 915th, and the 916th second of the whole road section; (c) the phase plane diagrams of $(\sigma, \partial \sigma / \partial x)$ on the 910th, 911th, the 912th, the 913th, the 914th, the 915th, and the 916th second of the whole road section.

diagram of $(v, \partial v / \partial x)$. It describes the velocity fluctuations at these moments on the whole road, but it does not highlight the congested traffic phenomena we are chiefly concerned about. However, the corresponding phase plane diagram of $(\sigma, \partial \sigma / \partial x)$ in Figure 5(c) obviously shows that the inner circle of the current time turns to the outer ring of the next second and the traffic become congested with time. So it clearly reflects unstable traffic phenomena.

Similarly, Figure 6 is the change curves of $v$ and $\sigma$ in the first 40 minutes on the $2 \mathrm{nd}$, the $3 \mathrm{rd}$, the 4 th, the 5 th, and the 6th road section. As you can see from Figure 6(a), all of the curves almost coincide together and we cannot see the velocity variation with section. Figure 6(b) describes the size and speed of the velocity variation with time but it cannot describe the jam traffic situation on different sections. Figure 6(c) shows that the outer ring of the current section turns to the inner circle of the next section and mainly reflects the degree of the congestion on different sections. The result is more obvious when the traffic system is more instable.

Figure 7 shows that the traffic jam becomes more serious when the amplitude of localized perturbation $\Delta \rho_{0}$ was increased to $0.15 \mathrm{veh} / \mathrm{m}$. Figure $7(\mathrm{a})$ is the phase plane diagrams of $(\rho, \partial \rho / \partial t)$ and it describes the variation of density with time when the section is fixed. We can see the values of density on some sections approach to the jam density $0.2 \mathrm{veh} / \mathrm{m}$ but the density change rates are all less than 0.005 in Figure 7(a). However, the change rates of $\eta$ in Figure 7(b) increase greatly and the maximal value is close to 0.5 , which are almost 100 times bigger than that of $\rho$ in Figure 7(a). The values of $\eta$ in Figure 7(b) also change sharply even when there is a very small density fluctuation in Figure $7(a)$. So the phase plane diagram of $(\eta, \partial \eta / \partial t)$ in Figure $7(b)$ emphasizes 


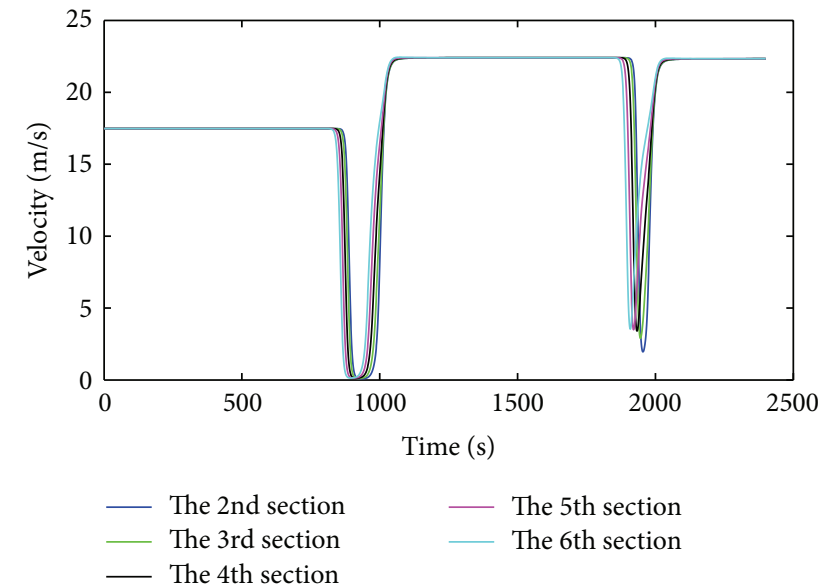

(a)

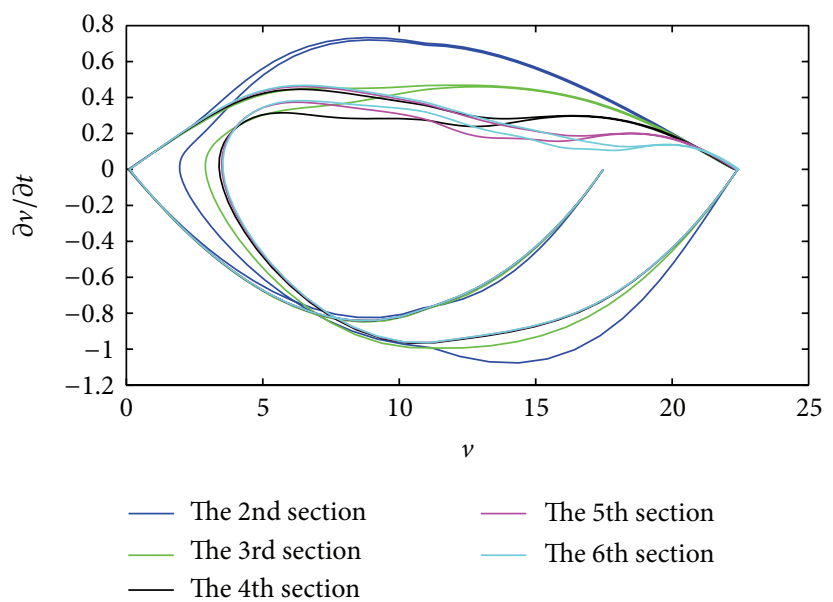

(b)

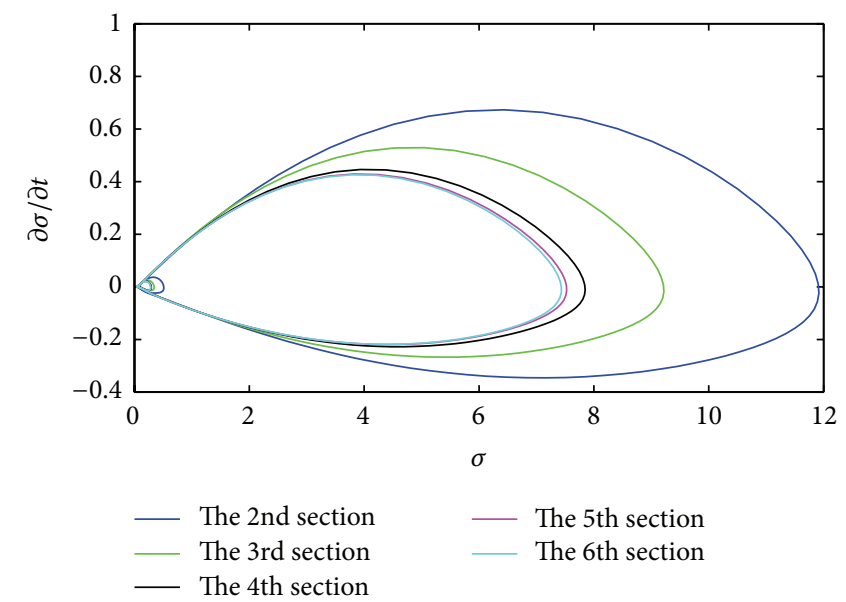

(c)

Figure 6: (a) Velocity-time variation curves on the $2 \mathrm{nd}$, the $3 \mathrm{rd}$, the 4th, the 5th, and the 6th road section; (b) the phase plane diagram of $(v, \partial v / \partial t)$ on the $2 \mathrm{nd}$, the $3 \mathrm{rd}$, the $4 \mathrm{th}$, the $5 \mathrm{th}$, and the 6 th road section; (c) the phase plane diagram of $(\sigma, \partial \sigma / \partial t)$ on the $2 \mathrm{nd}$, the $3 \mathrm{rd}$, the 4 th, the 5 th, and the 6 th road section.

the instable situation of the traffic system we are chiefly concerned about. When the vehicle density approaches congestion, the value of $\eta$ will increase greatly. The corresponding curves of such traffic jams accounted for a large proportion in Figure 7(b) while most small amplitude density fluctuations under stable traffic conditions just centered in a small area near the initial value. Likewise, Figures $7(\mathrm{c})$ and $7(\mathrm{~d})$ also illustrate it by the variation of $\rho$ and $\eta$ with sections.

Next we explored what the stable traffic will evolve into when the initial density was set to $0.08 \mathrm{veh} / \mathrm{m}$, and the results were compared with the density temporal evolution and phase plane diagrams as follows.

In Figure 8(a), the initial traffic flow density was set in the stable range, and hence the perturbation died out with time without any amplification. The traffic flow tended to be stable homogeneous state.

Figures $8(\mathrm{~b})$ and $8(\mathrm{c})$ are, respectively, the combinations of variation curves of $\eta$ and $\sigma$ on each road section during the first 40 minutes. It can be seen that all the curves vary from outer to inner and none of them tends to infinity when we find out the starting point and the trajectory of them. That meant the value of $\eta$ and $\sigma$ is decreasing and the initial small perturbation is dissipated with time. Moreover, the small density fluctuation in Figure 8(a) is amplified obviously in the phase plane diagrams. Further studies conclusively showed that all the curves of Figure 8 (b) gradually converge to the stable point $(8.3333,0)$ with time. It is consistent with the phenomena shown in Figure 8(a) that the traffic flow densities finally converge to the initial density. Figures 8 (d) and $8(\mathrm{e})$ consist of a number of circles and also describe the density and velocity fluctuations of the whole road per second. In contrast to the unstable situation, all the curves change over time from the outer circle to the inner ring. It is also demonstrated that density fluctuations of the whole road are gradually decreased with time. The initial small perturbation is dissipated with time. The whole traffic system is stable.

The variations of density with time on some concrete road sections were investigated in Figure 9. Figure 9(a) shows that the initial perturbations on different road sections are 


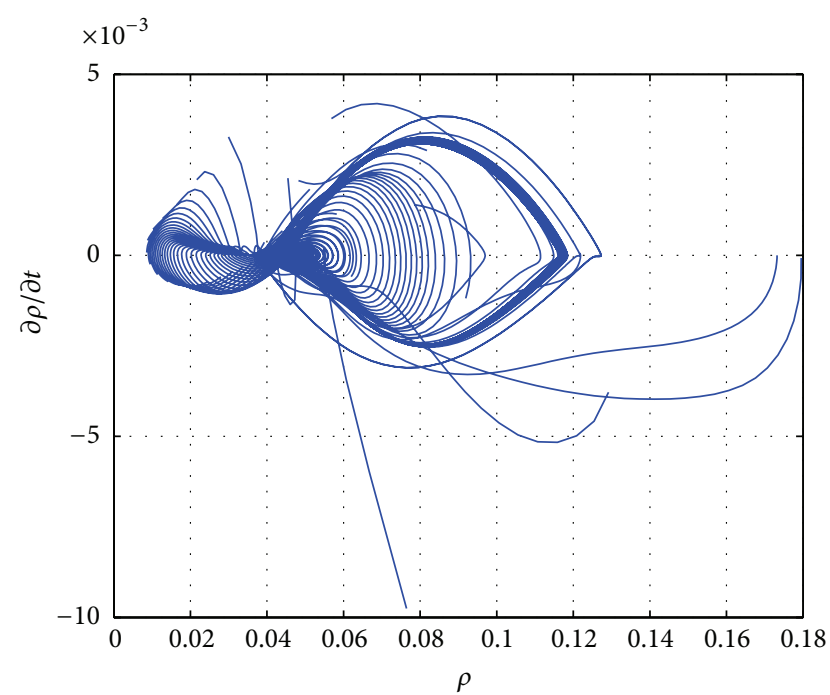

(a)

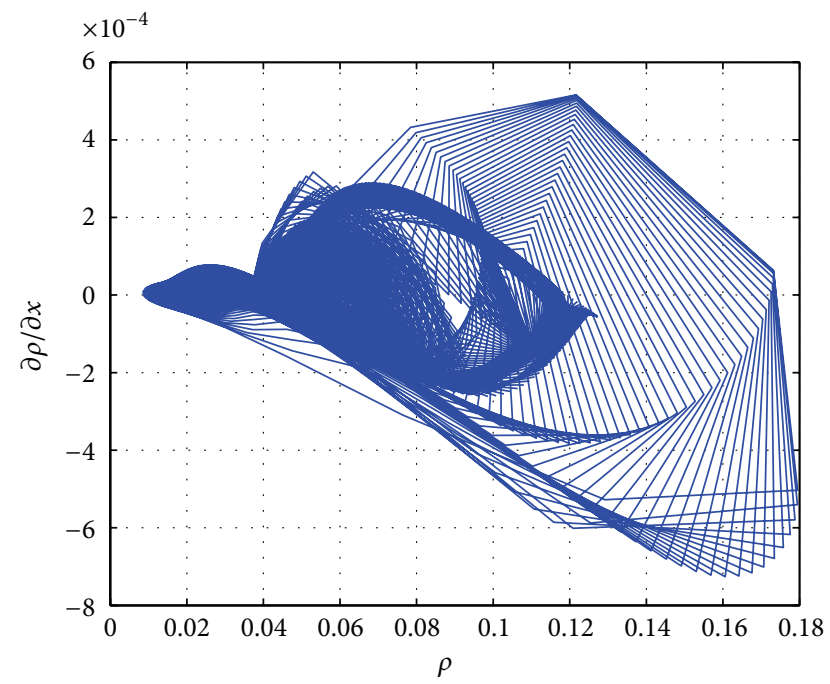

(c)

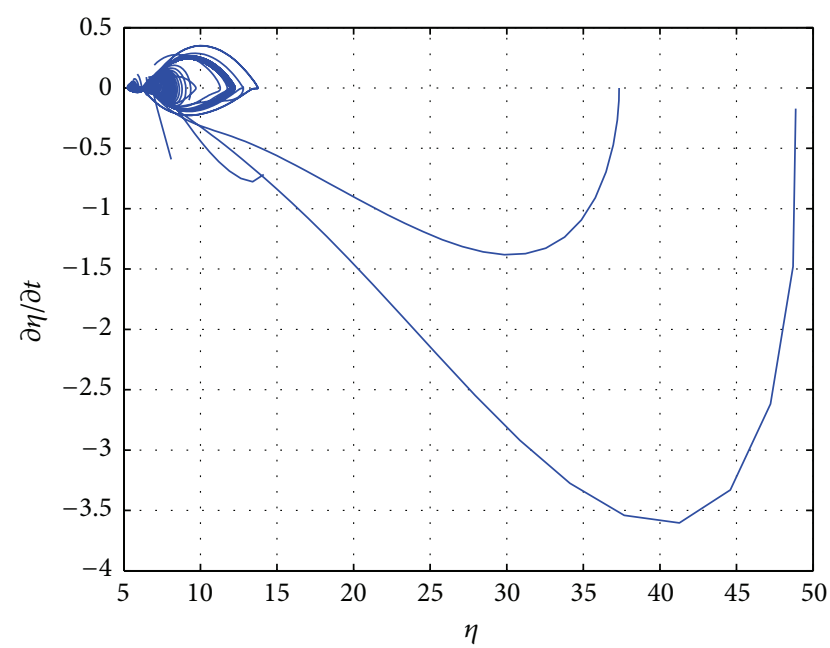

(b)

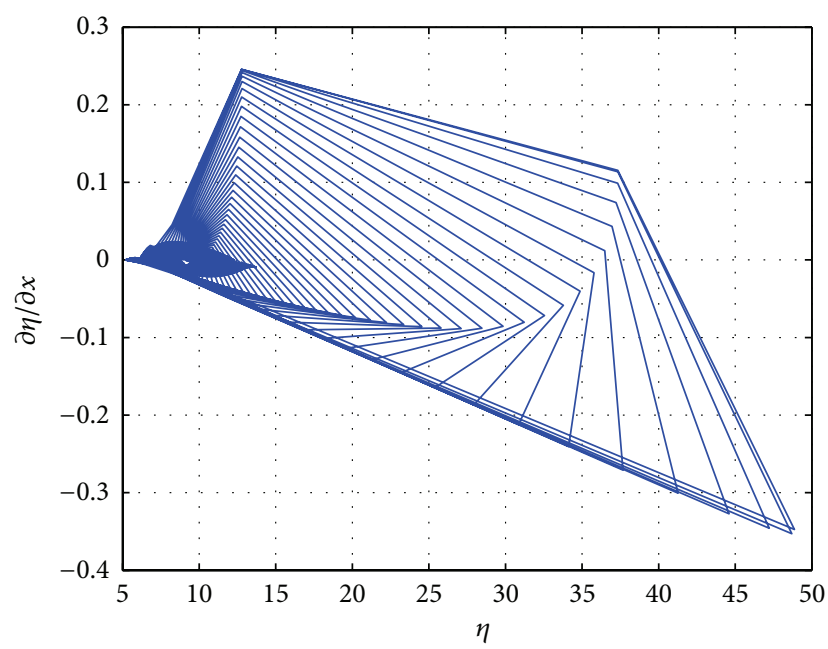

(d)

FIGURE 7: Traffic evolution with a homogeneous initial traffic $\rho_{0}=0.046 \mathrm{veh} / \mathrm{m}$ and a localized perturbation of amplitude $\Delta \rho_{0}=0.15 \mathrm{veh} / \mathrm{m}$ : (a) the phase plane diagram of $(\rho, \partial \rho / \partial t)$; (b) the phase plane diagram of $(\eta, \partial \eta / \partial t)$; (c) the phase plane diagram of $(\rho, \partial \rho / \partial x)$; (d) the phase plane diagram of $(\eta, \partial \eta / \partial x)$.

all dissipated with time and the density finally converges to the given initial value of $0.08 \mathrm{veh} / \mathrm{m}$. All the curves corresponding to these roads in Figure 9(b) change from the outer circle to the inner ring. It also illustrates that the perturbations spread upstream and are dissipated finally.

Comparing the phase plane diagrams with the temporal evolution of density shows that the stop-and-go traffic phenomenon described by the new method is consistent with that described by traditional method. But, unlike the previous temporal evolution of density, the phase plane diagram focuses on the density fluctuation we mainly care for and does not highlight the most homogeneous state of stable traffic flow. Moreover, it describes the variation of density or velocity with time or sections more clearly.

\section{Analysis of Shock and Rarefaction Waves Using the New Method}

In order to illustrate this new method further, we used the substituted model and phase plane diagrams to describe the well-known shock and rarefaction waves from a system stability perspective. The results are also compared with traditional temporal evolution of vehicle density based on the original traffic flow model.

We investigated how the traffic flow fronts between a congested and a nearly free traffic evolve with two Riemann initial conditions. These two initial conditions are

$$
\begin{aligned}
\rho_{u}^{1} & =0.04(\mathrm{veh} / \mathrm{m}), \\
\rho_{d}^{1} & =0.18(\mathrm{veh} / \mathrm{m}),
\end{aligned}
$$




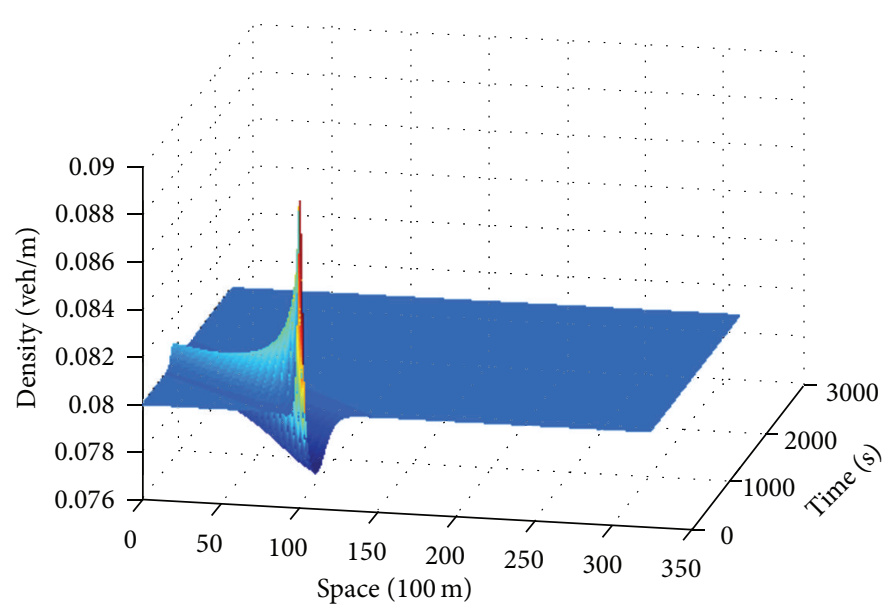

(a)

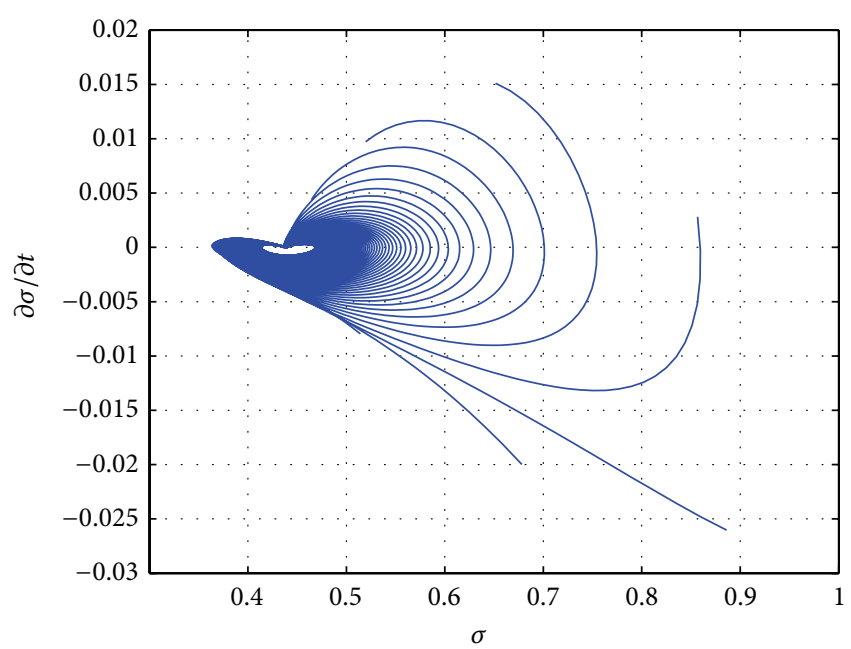

(c)

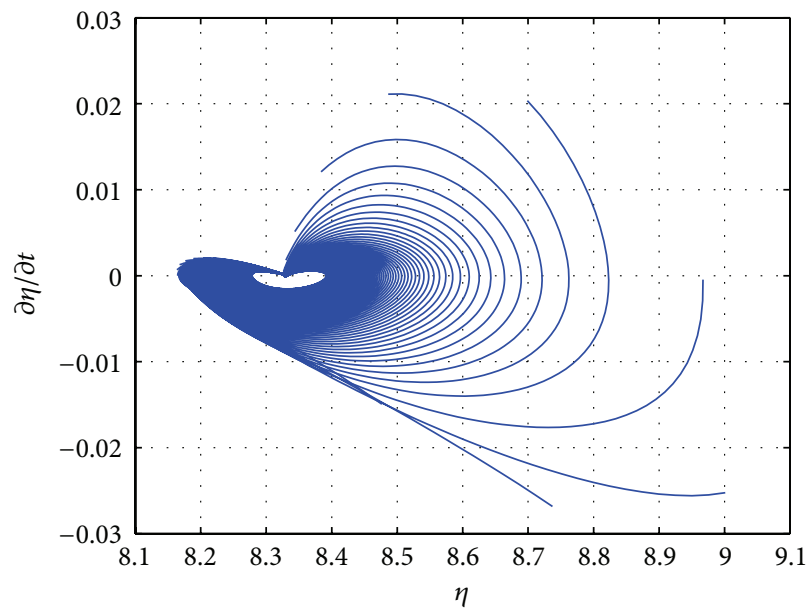

(b)

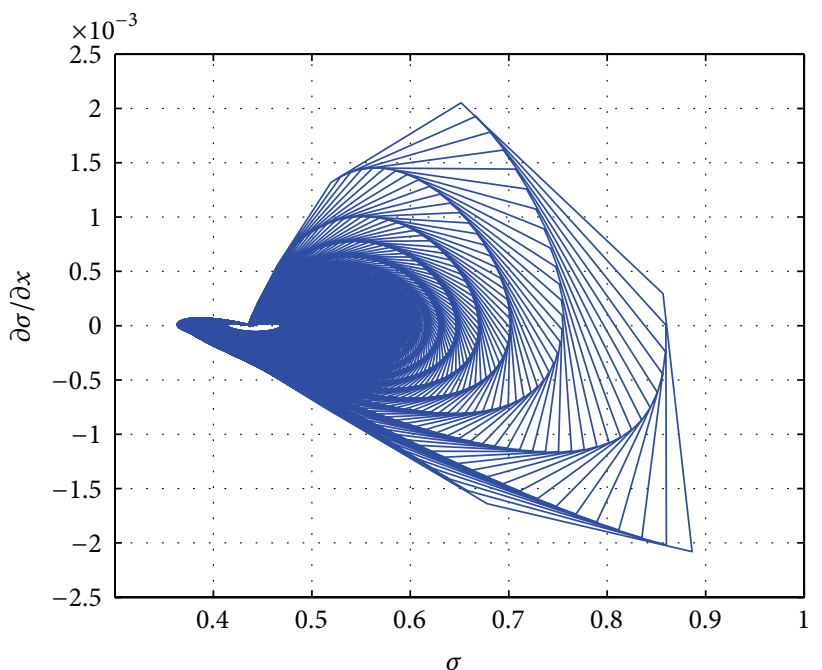

(d)

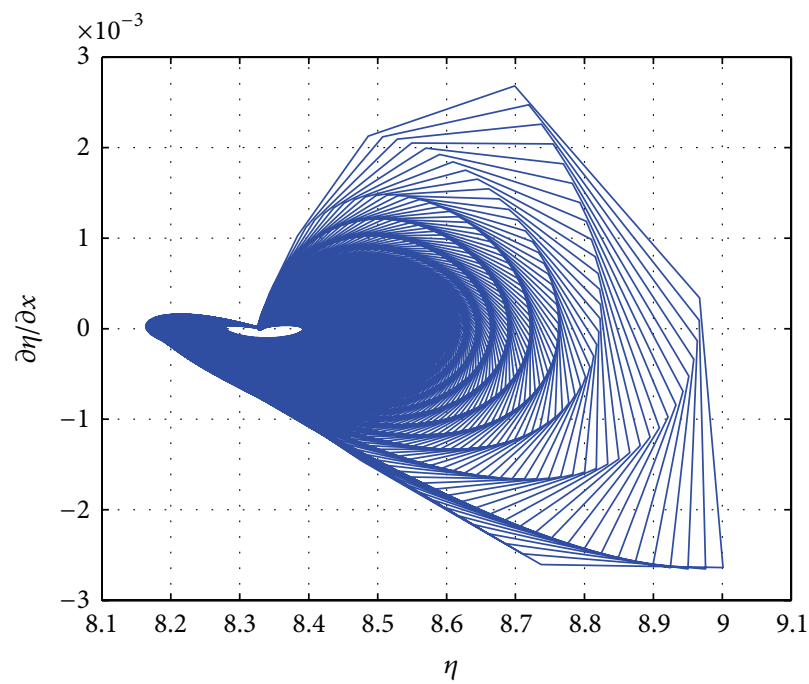

(e)

FIGURE 8: Evolution of small perturbation under the initial density $\rho_{0}=0.08 \mathrm{veh} / \mathrm{m}$ : (a) the temporal evolution of vehicle density; (b) the phase plane diagram of $(\eta, \partial \eta / \partial t)$; (c) the phase plane diagram of $(\sigma, \partial \sigma / \partial t)$; (d) the phase plane diagram of $(\sigma, \partial \sigma / \partial x)$; (e) the phase plane diagram of $(\eta, \partial \eta / \partial x)$. 


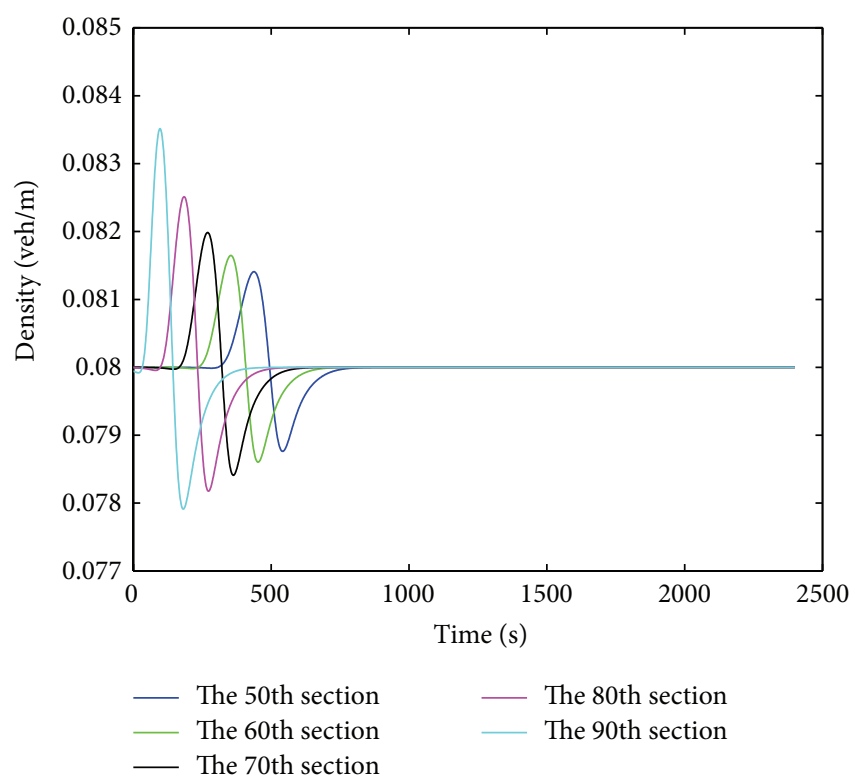

(a)

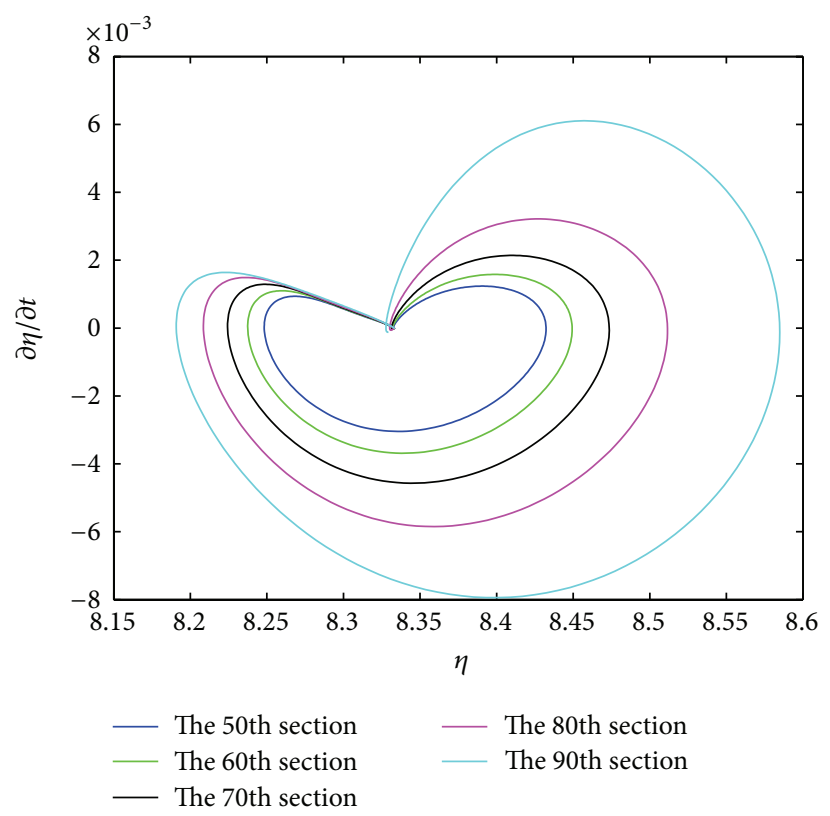

(b)

Figure 9: (a) Density-time variation curves on the fiftieth, the sixtieth, the seventieth, the eightieth, and the ninetieth road section; (b) the phase plane diagram of $(\eta, \partial \eta / \partial t)$ on the fiftieth, the sixtieth, the seventieth, the eightieth, and the ninetieth road section.

$$
\begin{aligned}
& \rho_{u}^{2}=0.18(\mathrm{veh} / \mathrm{m}), \\
& \rho_{d}^{2}=0.04(\mathrm{veh} / \mathrm{m}),
\end{aligned}
$$

where $\rho_{u}$ and $\rho_{d}$ are upstream and downstream densities, respectively. Condition (9a) corresponds to a shock wave situation, while condition (9b) corresponds to a rarefaction wave situation. Initial speed conditions are

$$
\begin{aligned}
& u_{u}^{1,2}=u_{e}\left(k_{u}^{1,2}\right), \\
& u_{d}^{1,2}=u_{e}\left(k_{d}^{1,2}\right) .
\end{aligned}
$$

Free boundary conditions are used here; that is, $\partial \rho / \partial x$ and $\partial u / \partial x$ are equal to zero on both sides. We have taken the test road section as $20 \mathrm{~km}$ long and it is divided into 100 meshes of equal length, the time interval is $1 \mathrm{~s}$. The related parameters of our model are as follows:

$$
\begin{aligned}
v_{f} & =30 \mathrm{~m} / \mathrm{s}, \\
\rho_{m} & =0.2 \mathrm{veh} / \mathrm{m}, \\
\tau & =10 \mathrm{~s}, \\
c_{m} & =c_{0}=11 \mathrm{~m} / \mathrm{s} .
\end{aligned}
$$

Figures 10 and 11 show, respectively, how the rarefaction wave front and shock wave front evolve under the Riemann initial conditions (9b) and (9a). By contrast, we first described the rarefaction wave phenomenon with the temporal development of density and velocity based on Jiang's model in Figures $10(\mathrm{a})$ and $10(\mathrm{~b})$. Then we made a cross-sectional analysis along the displacement axis of them to observe the variation of density and velocity with displacement when time is fixed. Using the substituted model, the change rule curves of $\eta$ and $\sigma$ per second on the whole road can be drawn in the phase plane diagrams in Figures 10(c) and 10(d). If we draw these curves one by one in the order of time, we can see all the curves change from one equilibrium point $(50,0)$ to another equilibrium point $(6.25,0)$. It is demonstrated that density gradually decreases from a high value of the upstream section toward a low value of the downstream section. Moreover, the change rate of curves decreases with time. It also illustrates that density varies more and more smoothly with time. We also found all the curves in Figure $10(\mathrm{~d})$ change from one equilibrium point $(0.8184,0)$ to another equilibrium point $(0.0346,0)$ if we draw them one by one in the order of time. It demonstrates that velocity gradually increases from a low value of congested upstream section toward a high value of nearly free-flow traffic on the downstream section. Moreover, the change rate of curves also decreases with time. It also illustrates that velocity varies more and more smoothly with time.

Figures 11(a) and 11(b) show how the shock wave front evolves with the temporal development of density and velocity based on Jiang's model. We also investigated the variation of density and velocity with displacement when time is fixed. Using the substituted model, the phase plane diagrams in Figures 11(c) and 11(d) depict the change of $\eta$ and $\sigma$ per second on the whole road. All curves also change from one equilibrium point to another, but the direction is in opposition to Figures $10(\mathrm{c})$ and $10(\mathrm{~d})$. It demonstrates that density gradually increases from a low value of the downstream section toward a high value of the upstream section. Moreover, the change rate of curves also decreases 


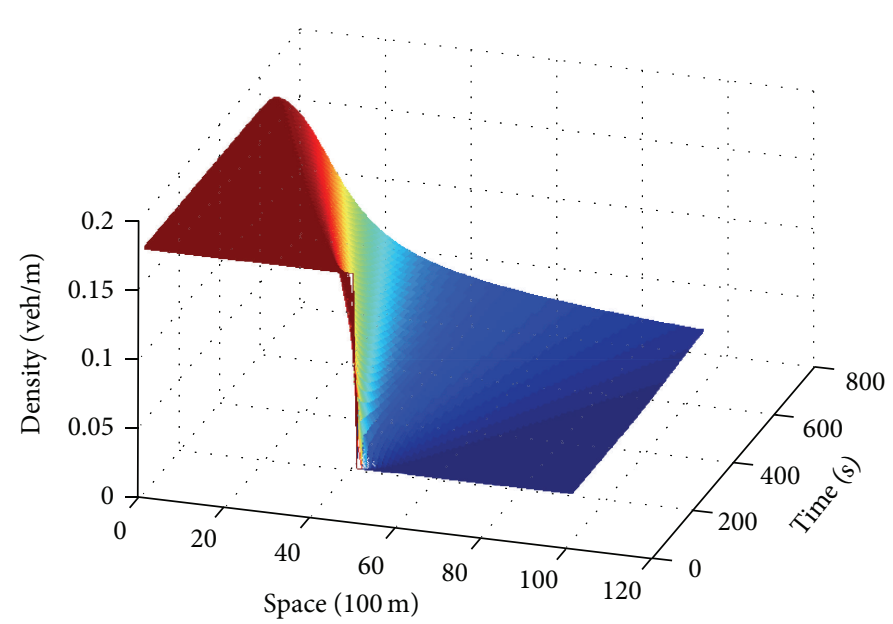

(a)

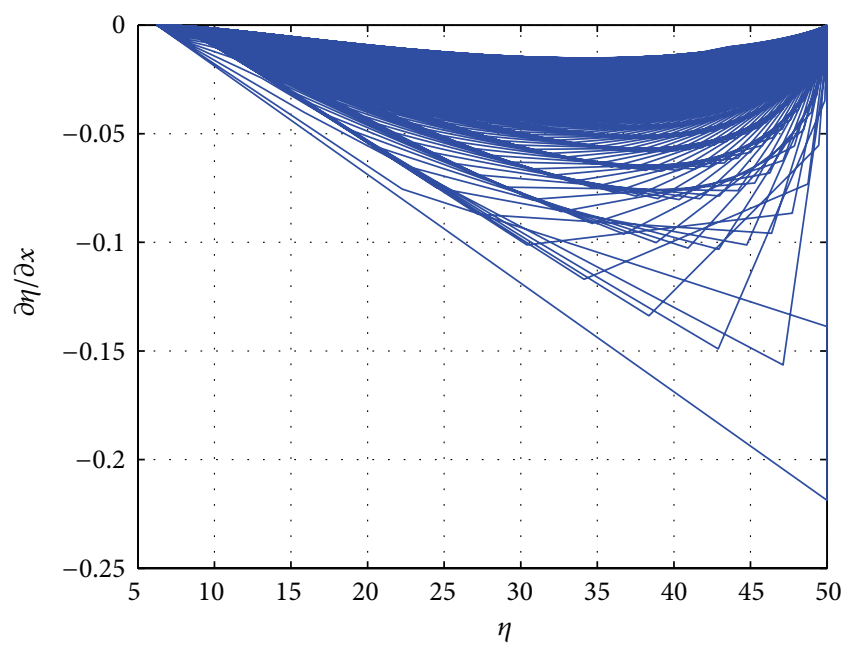

(c)

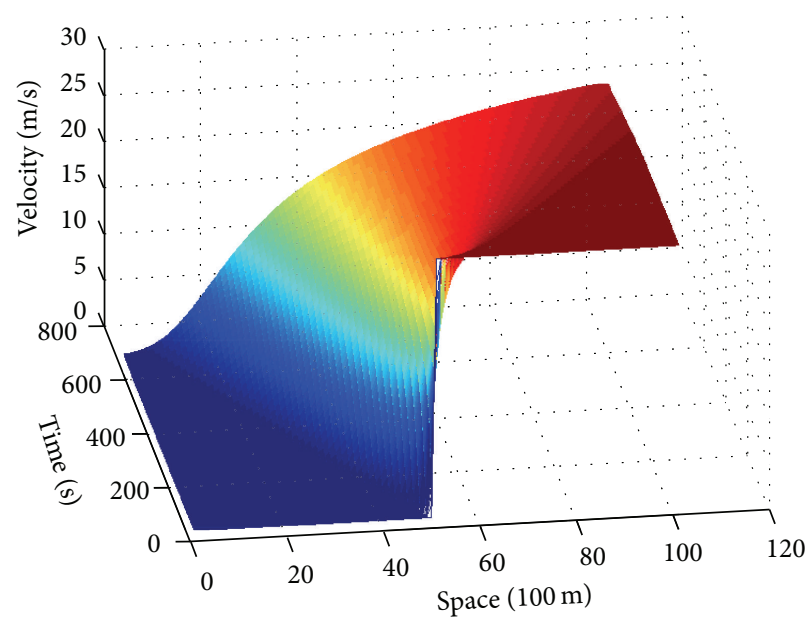

(b)

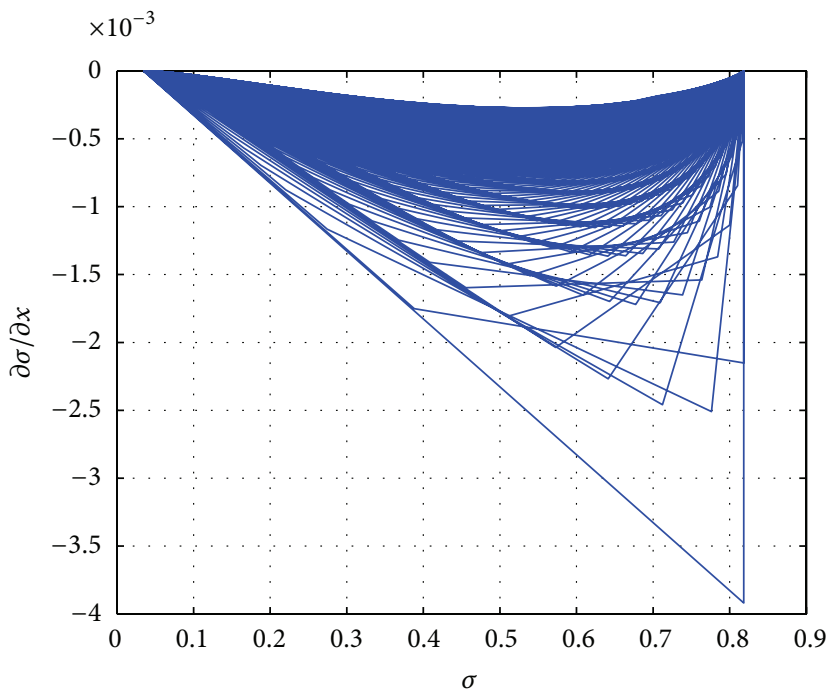

(d)

FIGURE 10: Rarefaction waves under the Riemann initial condition of (9b): (a) temporal development of density $\rho(x, t)$; (b) temporal development of speed $v(x, t)$; (c) the phase plane diagram of $(\eta, \partial \eta / \partial x)$; (d) the phase plane diagram of $(\sigma, \partial \sigma / \partial x)$.

with time. It also illustrates that the shock wave front is smoothed over time. So the phase plane diagram can describe the rarefaction wave and shock wave from a system stability perspective, which is consistent with the reality.

\section{Conclusions}

In this paper, we present a new method for analyzing the nonlinear traffic phenomena. This method makes use of variable substitution to transform a traditional traffic flow model into a new model. Using the new model the relationship between traffic congestion and the stability of the system can be obtained in the phase plane. So we can describe all kinds of nonlinear traffic phenomena with the phase plane diagrams and determine whether there will be traffic congestion or other abnormal phenomena from a global stability point. In order to specify this phase plane analysis method, we first build a new traffic flow model though substituting the variable in Jiang's model. Using the new model and the phase plane diagrams, we analyzed various traffic phenomena, such as the well-known shock waves, rarefaction waves, and stop-and-go. The results are consistent with that described by the original Jiang's model through the figures of temporal development of density. Moreover, unlike the previous temporal evolution of density, the phase plane diagrams highlight the unstable traffic phenomena we are chiefly concerned about. When the stop-and-go traffic appears and the traffic flow fluctuations tend to be unstable, the curves in phase plane diagrams are divergent and many of them tend to infinity. But when the fluctuations tend to be stable, all curves in phase plane diagrams change from outside to inside. When the shock wave and rarefaction wave appears, the curves in phase plane diagrams change from one equilibrium point to another. Moreover, the phase plane diagrams clearly reflect the size and speed of the density fluctuations. 


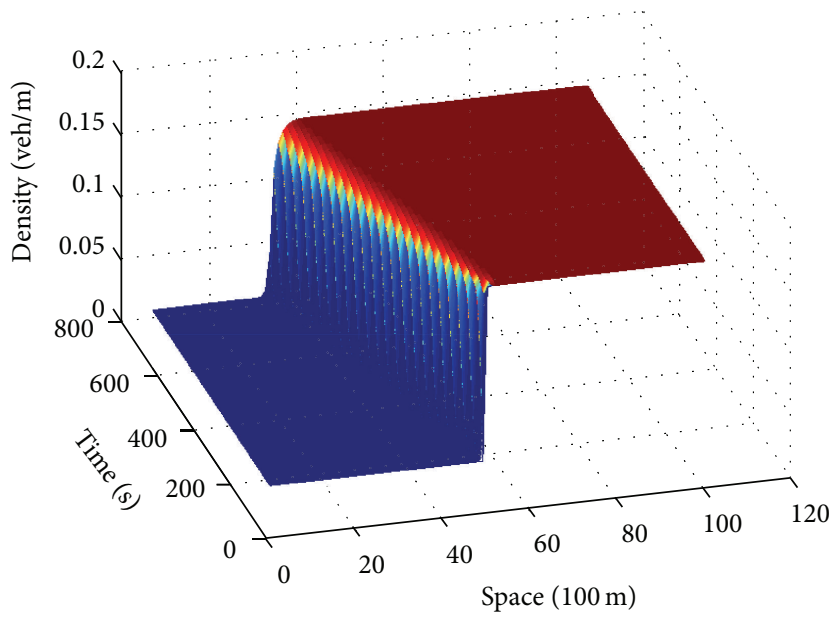

(a)

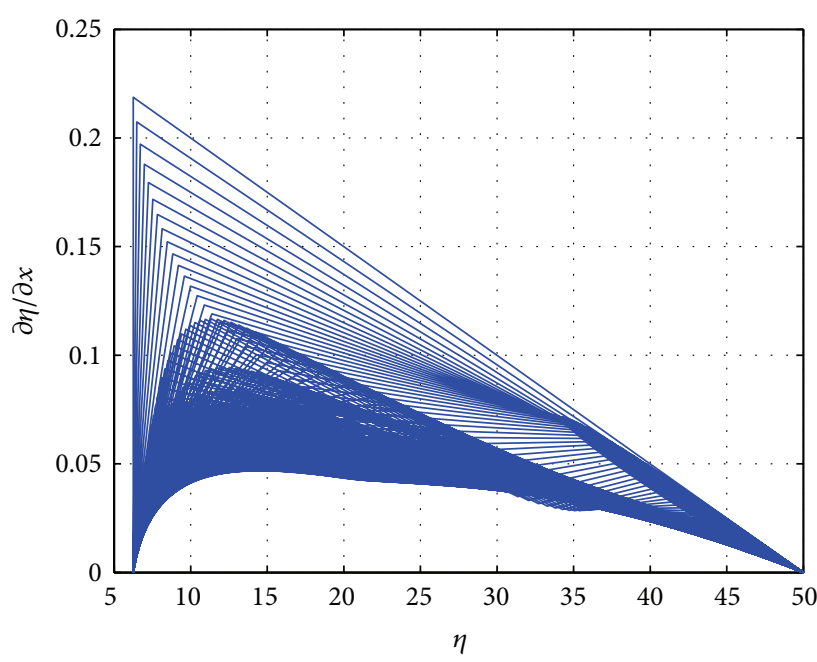

(c)

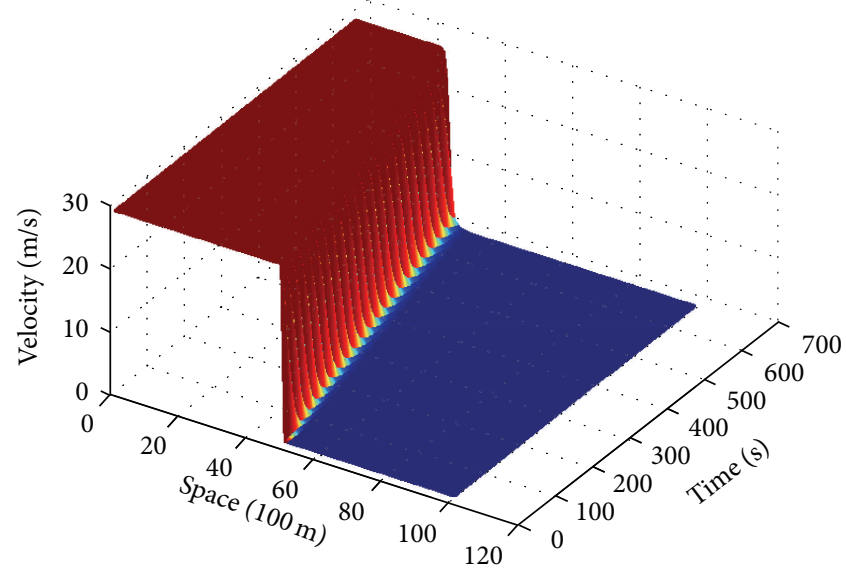

(b)

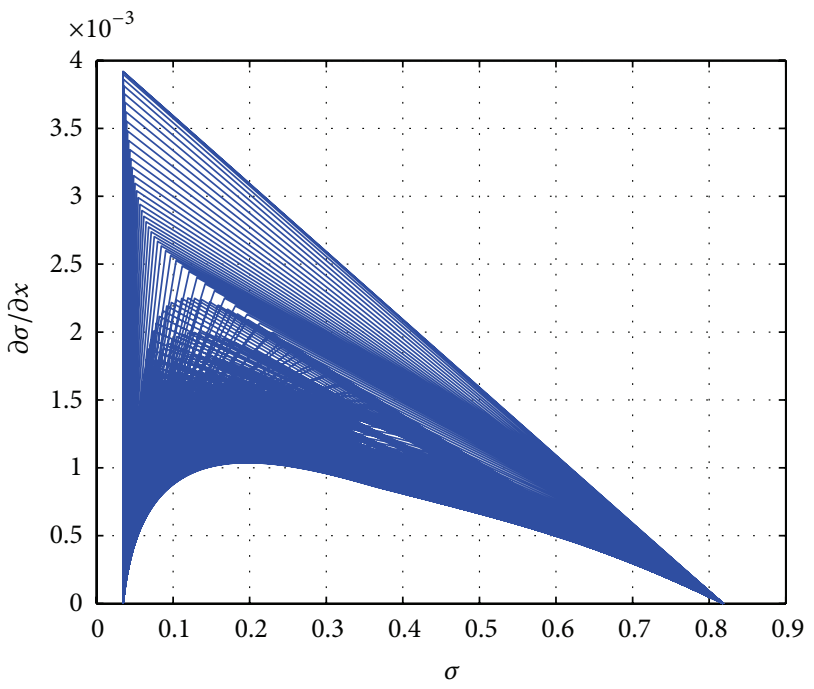

(d)

FIGURE 11: Shock waves under the Riemann initial condition of (9a): (a) temporal development of density $\rho(x, t)$; (b) temporal development of speed $v(x, t) ;(\mathrm{c})$ the phase plane diagram of $(\eta, \partial \eta / \partial x)$; (d) the phase plane diagram of $(\sigma, \partial \sigma / \partial x)$.

In the future work we will apply some mathematical tools such as branch and bound to the nonlinear stability analysis. It may be possible to apply some relative approaches of control theory to regulate the stability of traffic system through the equivalence relation between the traffic phenomena and system stability introduced in this paper.

\section{Conflict of Interests}

The authors declare that there is no conflict of interests regarding the publication of this paper.

\section{Acknowledgments}

The authors would like to thank the anonymous referees and the editor for their valuable opinions. This work is partially supported by the National Nature Science Foundation (NNSF) of China under Grant no. 61134004.

\section{References}

[1] A. K. Gupta and V. K. Katiyar, "Analyses of shock waves and jams in traffic flow," Journal of Physics A, vol. 38, no. 19, pp. 4069-4083, 2005.

[2] R. Jiang, Q.-S. Wu, and Z.-J. Zhu, "A new continuum model for traffic flow and numerical tests," Transportation Research Part B: Methodological, vol. 36, no. 5, pp. 405-419, 2002.

[3] L. Yu, T. Li, and Z.-K. Shi, "The effect of diffusion in a new viscous continuum traffic model," Physics Letters A: General, Atomic and Solid State Physics, vol. 374, no. 23, pp. 2346-2355, 2010.

[4] X. J. Ban, P. Hao, and Z. Sun, "Real time queue length estimation for signalized intersections using travel times from mobile sensors," Transportation Research Part C: Emerging Technologies, vol. 19, no. 6, pp. 1133-1156, 2011.

[5] H. J. Payne, "Models of freeway traffic and control," in Mathematical Models of Public Systems, vol. 1, pp. 51-61, Simulation Councils, 1971. 
[6] B. S. Kerner and P. Konhäuser, "Structure and parameters of clusters in traffic flow," Physical Review E, vol. 50, no. 1, pp. 5483, 1994.

[7] H. M. Zhang and W. Shen, "Numerical investigation of stopand-go traffic patterns upstream of freeway lane drop," Transportation Research Record, no. 2124, pp. 3-17, 2009.

[8] H. X. Ge and X. L. Han, "Density viscous continuum traffic flow model," Physica A: Statistical Mechanics and Its Applications, vol. 371, no. 2, pp. 667-673, 2006.

[9] L. Yu, T. Li, and Z.-K. Shi, "The effect of diffusion in a new viscous continuum traffic model," Physics Letters A, vol. 374, no. 23, pp. 2346-2355, 2010.

[10] Z.-K. Shi and Y. Qiao, "Detection method of queuing vehicles on urban road," Journal of Traffic and Transportation Engineering, vol. 12, no. 5, pp. 100-109, 2012.

[11] E. I. Vlahogianni and M. G. Karlaftis, "Comparing traffic flow time-series under fine and adverse weather conditions using recurrence-based complexity measures," Nonlinear Dynamics, vol. 69, no. 4, pp. 1949-1963, 2012.

[12] H. Gong and W. Jin, "Analysis of urban car owners commute mode choice based on evolutionary game model," Journal of Control Science and Engineering, vol. 2015, Article ID 291363, 5 pages, 2015.

[13] M. J. Lighthill and G. B. Whitham, "On kinematic waves. II. A theory of traffic flow on long crowded roads," Proceedings of the Royal Society of London. Series A. Mathematical, Physical and Engineering Sciences, vol. 229, pp. 317-345, 1955.

[14] P. I. Richards, "Shock waves on the highway," Operations Research, vol. 4, pp. 42-51, 1956.

[15] T. Q. Tang, H. J. Huang, and H. Y. Shang, "A new macro model for traffic flow with the consideration of the driver's forecast effect," Physics Letters, Section A: General, Atomic and Solid State Physics, vol. 374, no. 15-16, pp. 1668-1672, 2010.

[16] G. H. Peng, W. Song, Y. J. Peng, and S. H. Wang, "A novel macro model of traffic flow with the consideration of anticipation optimal velocity," Physica A: Statistical Mechanics and Its Applications, vol. 398, pp. 76-82, 2014.

[17] R. E. Chandler, R. Herman, and E. W. Montroll, "Traffic dynamics: studies in car following," Operations Research, vol. 6, pp. 165-184, 1958.

[18] L. A. Pipes, "An operational analysis of traffic dynamics," Journal of Applied Physics, vol. 24, pp. 274-281, 1953.

[19] X.-J. Wang, X.-M. Xi, and G.-F. Gao, "Reinforcement learning ramp metering without complete information," Journal of Control Science and Engineering, vol. 2012, Article ID 208456, 8 pages, 2012.

[20] J. Zhou, Z. K. Shi, and J. L. Cao, "An extended traffic flow model on a gradient highway with the consideration of the relative velocity," Nonlinear Dynamics, vol. 78, no. 3, pp. 1765-1779, 2014.

[21] T. Q. Tang, W. F. Shi, H. Y. Shang, and Y. P. Wang, "A new car-following model with consideration of inter-vehicle communication," Nonlinear Dynamics, vol. 76, pp. 2017-2023, 2014.

[22] Y. Hu, T. Ma, and J. Chen, "An extended multi-anticipative delay model of traffic flow," Communications in Nonlinear Science and Numerical Simulation, vol. 19, no. 9, pp. 3128-3135, 2014. 

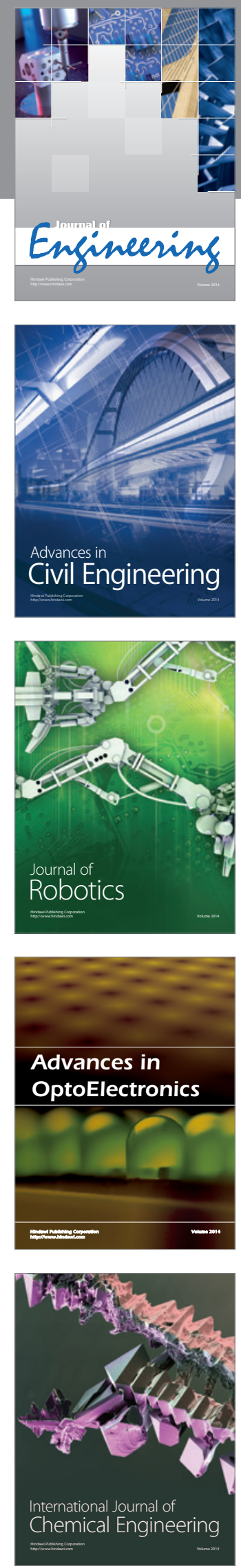

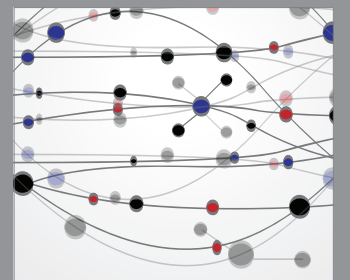

The Scientific World Journal
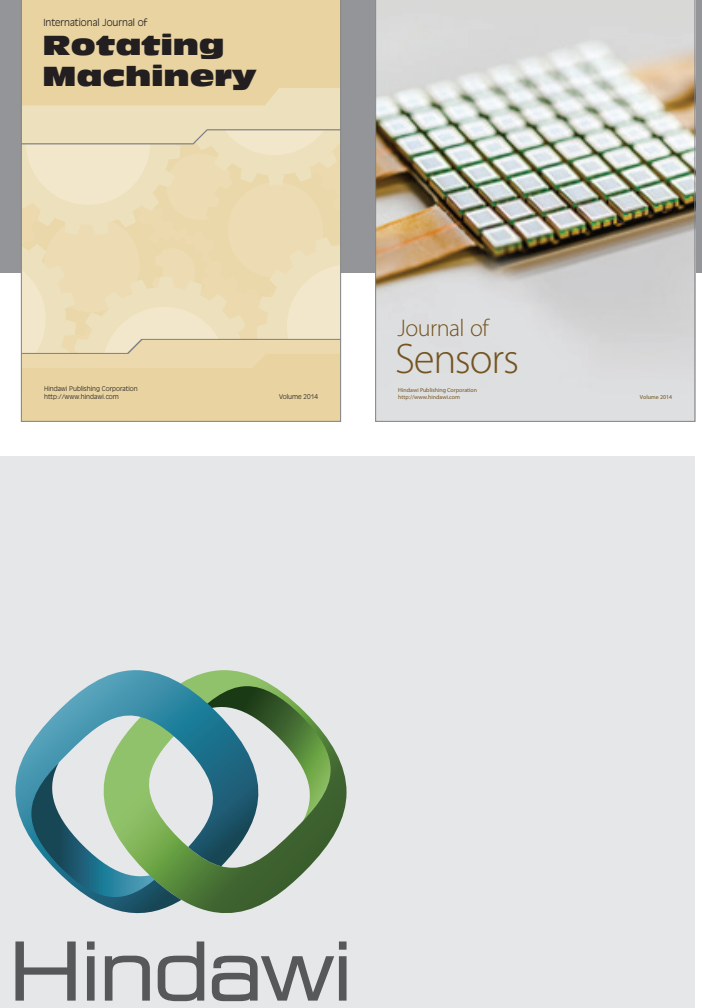

Submit your manuscripts at http://www.hindawi.com
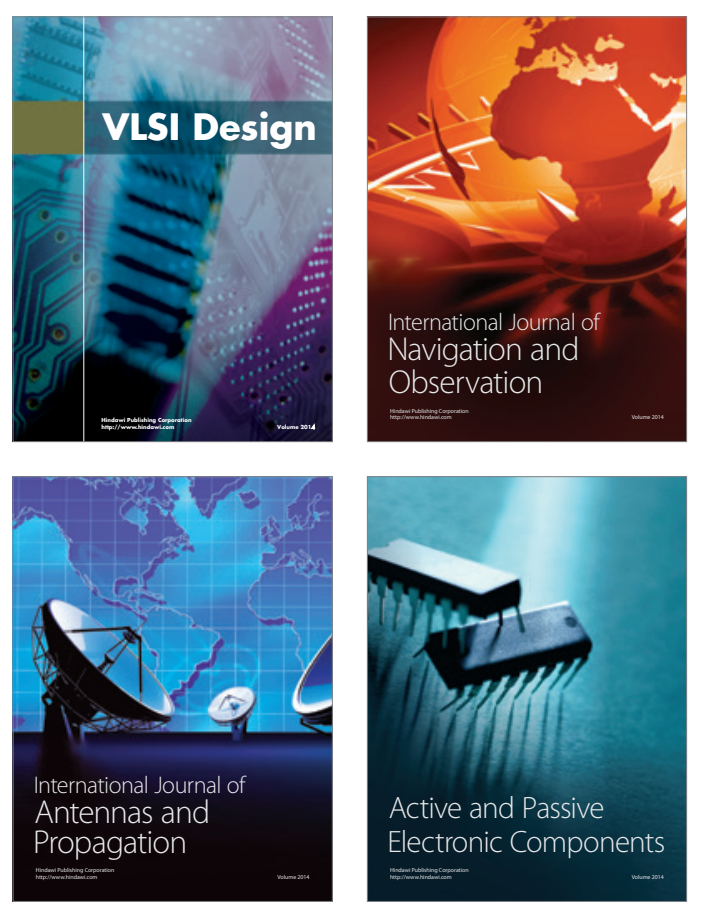
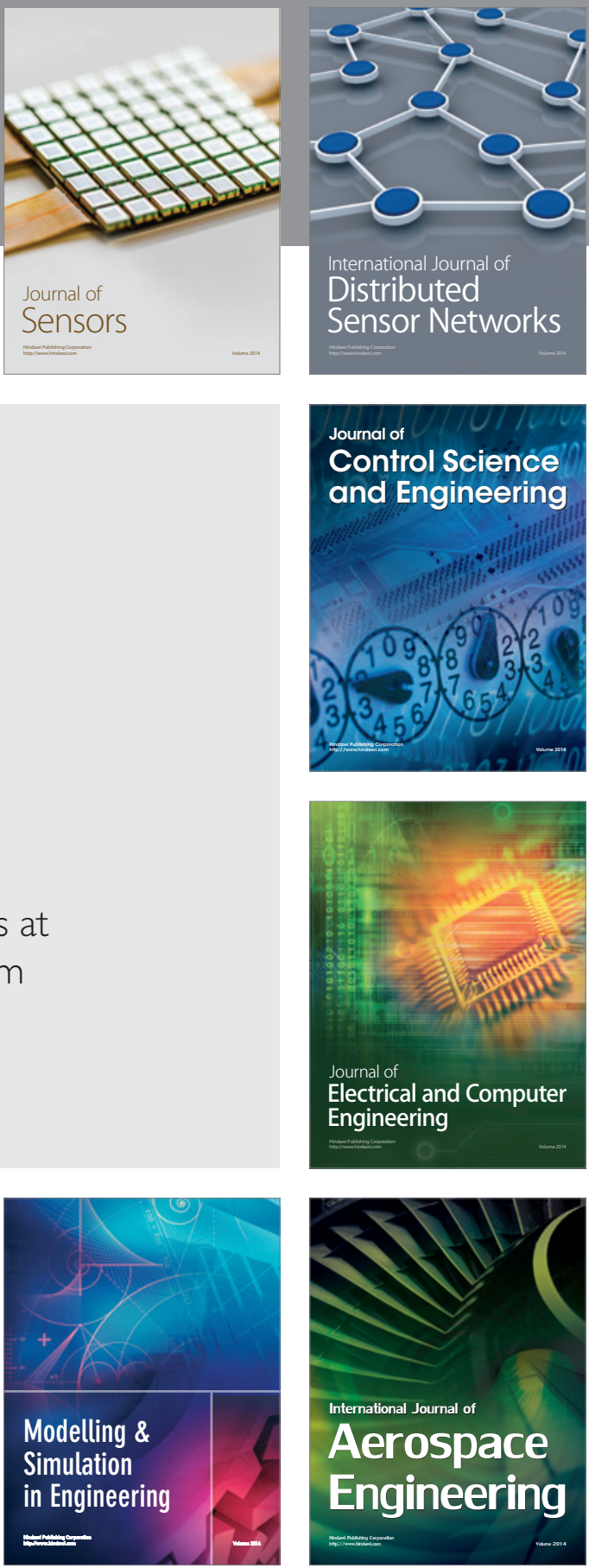

Journal of

Control Science

and Engineering
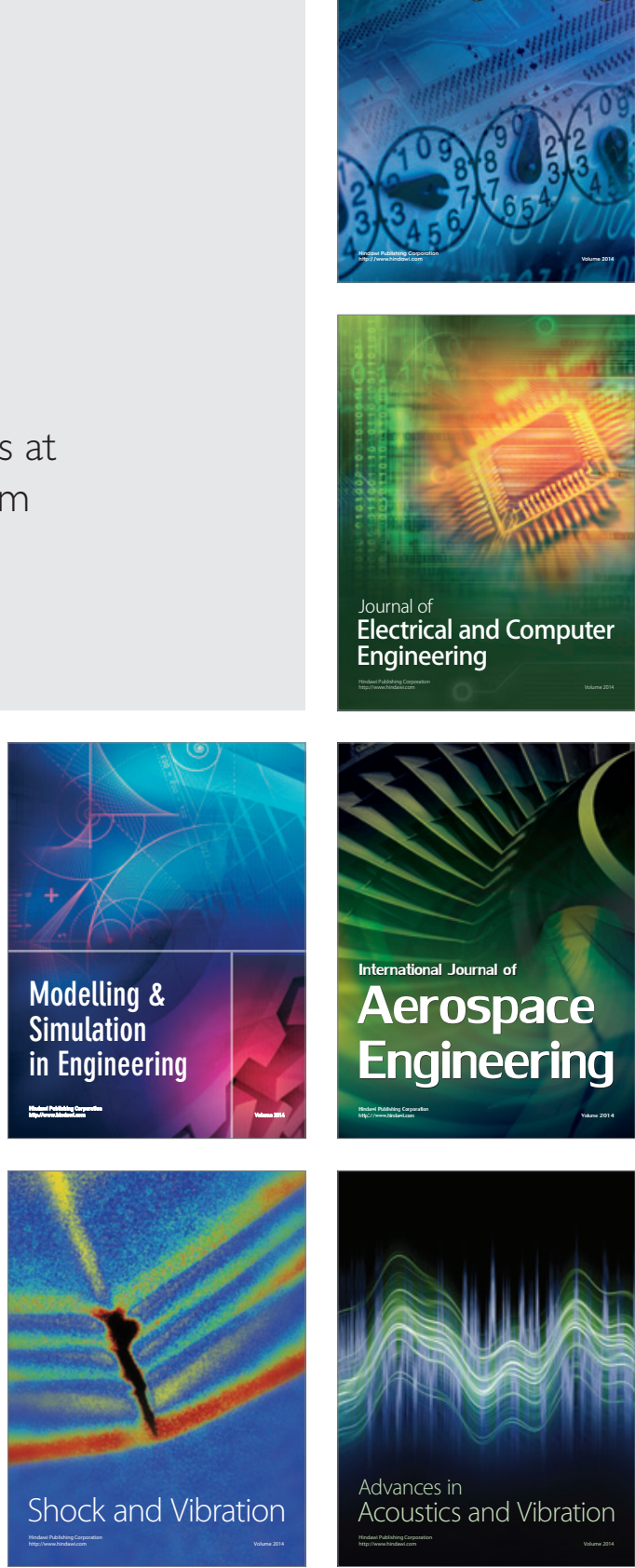